\title{
Central-Moments-Based Lattice Boltzmann for Associating Fluids: A New Integrated Approach
}

DOI:

10.1021/acs.jpcb.9b10989

\section{Document Version}

Accepted author manuscript

Link to publication record in Manchester Research Explorer

\section{Citation for published version (APA):}

Asadi, M. B., De Rosis, A., \& Zendehboudi, S. (2020). Central-Moments-Based Lattice Boltzmann for Associating Fluids: A New Integrated Approach. The Journal of Physical Chemistry B, 124(14), 2900-2913.

https://doi.org/10.1021/acs.jpcb.9b10989

\section{Published in:}

The Journal of Physical Chemistry B

\section{Citing this paper}

Please note that where the full-text provided on Manchester Research Explorer is the Author Accepted Manuscript or Proof version this may differ from the final Published version. If citing, it is advised that you check and use the publisher's definitive version.

\section{General rights}

Copyright and moral rights for the publications made accessible in the Research Explorer are retained by the authors and/or other copyright owners and it is a condition of accessing publications that users recognise and abide by the legal requirements associated with these rights.

\section{Takedown policy}

If you believe that this document breaches copyright please refer to the University of Manchester's Takedown Procedures [http://man.ac.uk/04Y6Bo] or contact uml.scholarlycommunications@manchester.ac.uk providing relevant details, so we can investigate your claim.

\section{OPEN ACCESS}




\title{
Central-Moments-Based Lattice Boltzmann for Associating Fluids: A New Integrated Approach
}

\author{
Mohammad Bagher Asadi ${ }^{1}$, Alessandro De Rosis ${ }^{2}$, Sohrab Zendehboudi ${ }^{*}$ \\ ${ }^{1}$ Faculty of Engineering and Applied Science, Memorial University, St. John's, NL, Canada \\ ${ }^{2}$ Department of Mechanical, Aerospace and Civil Engineering, The University of Manchester, Manchester M13 \\ 9PL, UK
}

\begin{abstract}
Dynamic and thermodynamic behavior of associating fluids play a crucial role in a variety of engineering and science disciplines. Cubic plus association equation of state (CPA EOS) is implemented in a central-moments-based lattice Boltzmann method (LBM) in order to mimic the thermodynamic behavior of associating fluids. The pseudopotential approach is selected to model the multiphase thermodynamic characteristics such as reduced density of associating fluids. The priority of central moments-based approach over multiple-relaxation-time collision operator is shown by performing double shear layers. The integration of central-moments-based LBM and CPA EOS is useful to simulate associating fluids at high flow rate conditions, which is extended to high-density ratio scenarios by increasing the anisotropy order of gradient operator. In order to increase the stability of the model, a higher anisotropy order of the gradient operator is implemented; about 34 present reduction in spurious velocities is noticed in some cases. The type of gradient operator considerably affects the model thermodynamic consistency. Finally, the model is validated by observing a straight line in the Laplace law test. Prediction of thermodynamic behaviours of associating fluids is of significance in biological processes as well as fluid flow in porous media.
\end{abstract}

Keywords: Associating fluid; Cubic plus association equation of state; central-moments-based lattice Boltzmann method; gradient operator; Laplace law

\section{INTRODUCTION}

Lattice Boltzmann method (LBM) has roots in the lattice gas automaton (LGA) ${ }^{1-4}$. This method has been developed as an option to simulate fluid flow behaviours and numerically solve nonlinear

\footnotetext{
* Corresponding Author's Email Address: szendehboudi@mun.ca
} 
equations. Basically, fluid dynamics can involve the motion of distributions/populations of fabricated particles which can stream and colloid along a Cartesian lattice. LBM has been employed in a broad range of engineering applications such as single-phase flow ${ }^{5-7}$, multiphase flow ${ }^{8-11}$, phase-change heat transfer ${ }^{10,12}$, turbulent regime in various transport phenomena ${ }^{13-17}$, and solving nonlinear partial differential equations (NPDEs) including convection-diffusion equations ${ }^{18-24}$. The kinetic basis of LBM makes it a powerful tool in the modeling of interfacial phenomena in the multiphase flow systems ${ }^{25-27}$. Although the origin of LBM is molecular dynamic kinetic which is more fundamental compared to the continuum approaches, it is capable of recovering the traditional macroscopic scale continuity and Navier-Stokes $(\mathrm{N}-\mathrm{S})$ equations. In the absence of required meshes movement, it can be parallelized due to locality of most of the computations. In the LBM, different boundary conditions can be handled easily.

As a common collision operator, Bhatnagar Gross and Krook (BGK), which is known as a single relaxation time collision operator, enforces all the populations to relax to an equilibrium state with the same rate ${ }^{28}$. Due to non-hydrodynamic ghost modes, the single relaxation time is instable in the high-velocity gradient cases, when the single relaxation time of BGK collision operator is implemented. Huang et al. ${ }^{29}$ demonstrated an inverse relationship between the highest achievable density (by aforementioned techniques) and the viscosity of fluid. In order to address this concern and achieve high Reynolds numbers, multiple-relaxation-time (MRT) collision operator is presented based on decomposing the collision operator as well as raw moments. These moments correspond to different hydrodynamic parameters and their fluxes which are able to relax at different time scales. The Prandtl number is fixed in BGK collision operator; by damping nonhydrodynamic modes ${ }^{30}$ and acoustic waves ${ }^{31}$ in MRT collision operator, one can achieve a model to adjust Prandtl number, improve the numerical stability, and handle the modeling of complex fluids such as viscoelastic fluids ${ }^{32}$. Since the MRT operator represents the collision in a frame at rest, the Galilean invariance is also trimmed. Yu and Fan utilized the MRT collision operator in the pseudopotential LBM ${ }^{33}$. Later on, this approach was modified through implementing an improved force scheme by $\mathrm{Li}$ et al. ${ }^{34}$.

Recently, Geier et al. ${ }^{35}$ introduced a novel collision kernel through shifting the lattice directions by the local fluid velocity. They presented a pyramidal hierarchical structure by developing the central moments (CMs) concept. In this structure, the post-collision state of a certain moment at a given order depends on lower-order ones. Thus, this collision operator is also known as "cascaded 
operator". Originally, an orthogonal basis of central moments, which relaxes to the equilibrium state of the continuous Maxwellian distribution is assumed for the cascaded LBM. In spite of overwhelming analytical formulation and practical implementation specifically in threedimensional cases ${ }^{36}$, this method leads to a considerable stability enhancement in many cases ${ }^{35}$, 37-54. Recently, De Rosis developed a new LBM framework based on the central moments, which is different from the cascaded scheme in two aspects. First, a non-orthogonal basis is adopted and second, the relaxation to the discrete second-order shortened the equilibrium population. The nonpyramidal structure demonstrates notable characteristics in terms of stability, convergence, and accuracy ${ }^{55-56}$.

Generally, two-phase/multiphase LBMs can be categorized in four groups: color-gradient ${ }^{57-58}$, pseudopotential ${ }^{59-60}$, free-energy ${ }^{61-62}$, and mean-field ${ }^{63}$. However, the LBM classification can be changed based on different aspects such as methodology, structure, limitations and capabilities, and application ${ }^{10}$. Due to the simplicity in the implementation and good efficiency in the computation, the pseudopotential LBM approach has received significant attention among several multiphase LBM models ${ }^{57-62,64}$. The particle interactions in the pseudopotential approach help the phase segregation so that this technique does not require other numerical methods to track and capture the interfaces. Besides the important features of the pseudopotential LBM, its shortcomings include thermodynamic inconsistency and high spurious velocities. Implementing the pseudopotential approach for reaching high Reynolds number/ low viscosity cases is still challenging as multiple issues (reaching high-density ratio, increasing thermodynamic consistency, decreasing spurious velocity, and increasing the Reynolds number/decreasing viscosity) should be handled simultaneously. Thermodynamic inconsistency is addressed by introducing new pseudopotential forms ${ }^{65}$ and effective forcing scheme ${ }^{66}$. Sbragaglia et al. introduced multi-range pseudopotential through combination of nearest and next-nearest neighbor interactions ${ }^{67}$. Falcucci et al. presented a double attractive pseudopotential to reach high-density ratios ${ }^{68}$. To overcome high spurious velocities, using higher anisotropy order of gradient operator is proposed ${ }^{69}$.

Yuan and Schaefer evaluated the thermodynamic consistency of LBM by comparing the simulation results of an stationry droplet with Maxwell equal-area construction when different cubic and non-cubic equations of state (EOSs) are used. Also, they investigated the impacts of different EOSs on the maximum sperious velocity ${ }^{70}$. They compared the LBM results with 
experimental data of water. In the liquid phase branch, a notable difference between the experimental data and LBM results was observed. Cubic EOSs such as Peng-Robinson (PR) ${ }^{71}$ and Soave-Redlich-Kwong (SRK) ${ }^{72}$ have been widely used to study the thermodynamic behaviors of hydrocarbon and non-hydrocarbon fluids due to their simplicity and accuracy. Compared to the physical interactions, polar components may form a stronger bonding. In the presence of fluids with hydrogen bonding, some unusual thermodynamic behaviors such as high liquid density, reverse density trend during solidification, and high boiling point are observed, which may not be fully explored (and justified) by cubic EOSs ${ }^{73}$. Water is an associating component which can exist in different fluid mixtures such as petroleum reservoir fluids in various forms such as connate water and injection fluids. Taking another example of associating fluids, asphaltene that might contain a group of complex components may form aggregates due to the association forces. Asadi and Zendehboudi ${ }^{74}$ introduced a new approach to decrease the differences between LBM results and experimental data of associating fluids. The cubic plus association (CPA) EOS was incorporated in LBM while the CPA EOS association parameters are recalculated to be used in LBM. Originally, CPA EOS was introduced by Kontogeorgis et al. ${ }^{75}$ to consider both physical and associating interactions. In general, CPA EOS consists of two terms to take into account both interactions. CPA EOS can turn to a cubic EOS in the absence of associating interactions. Although there are other EOSs such as perturbed-chain statistical associating fluid theory (PC-SAFT) with the capability of considering association bonds, CPA EOS has a lower computational cost.

Most of the available studies use the BGK collision operator to investigate the thermodynamic behaviors of fluids. As mentioned earlier, the single relaxation time collision operator can lead to valid/reasonable results within a specific range of dynamic viscosity. On the other hand, the MRT collision operator effectively works within limited intervals of Reynolds and Mach numbers.

In this paper, we aim to fill the above knowledge gap by implementing the CPA EOS in the central moments-based LBM, in order to improve the thermodynamical behaviors of fluids and decrease the numerical instability by increasing the isotropy order of gradient operator. The central moments-based collision operatore is employed to increase the stability of model at high Reynolds and Mach number conditions. CPA EOS is incorporated in the LBM to mime the thermodynamical behaviours of associatiog fluids.

The remaining of the paper is organized as follows. First, a review on pseudopotential LBM with different collision operators and incorporation of CPA EOS in the LBM are presented in the 
THEORY AND METHODOLOGY section. Then, the necessity of more accurate EOSs is presented by showing the flexibility of some common pseudopotentials to predict the thermodynamic properties. After that, the introduced approach is evaluated by comparing the twophase density results of central-moments-based LBM with Maxwell equal-area construction where different anisotropy orders of gradient operator are employed. The sperious velocity at different viscosity is examined, and the method is validated by Laplace law. Finally, the main outcomes of the current study are presented in the CONCLUSIONS section.

\section{THEORY AND METHODOLOGY}

In this section, first, the pseudopotential LBM, classical BGK collision operator, multi-relaxation time (MRT), and central-moments-based LBM are briefly explained. Then, the combination procedure of consistent pseudopotential interactions proposed by Shan and Chen (SC), Sbragaglia and Shan (SS), and cubic/CPA EOSs are presented.

Pseudopotential LBM, classical BGK, MRT, and central-moments based LBM. The discrete LB equation describes the evolution of the particle distribution function (PDF) $f_{i}$ in time $t$ and space $x=[x, y]$ which moves on a lattice structure along a direction $i$ with velocity $c_{i}=\left[c_{x i}, c_{y i}\right]$ during the time step, $\Delta t=1$. The corresponding equation is presented as follows:

$$
f_{i}\left(\boldsymbol{x}+\Delta t \boldsymbol{c}_{\boldsymbol{i}}, t+\Delta t\right)=f_{i}(\boldsymbol{x}, t)+\Omega_{i}(\boldsymbol{x}, t)+S_{i}
$$

In Equation (1), $S_{i}$ stands for the forcing term in the discrete LB equation. Equation (1) is solved through the following steps:

$$
\begin{array}{cl}
\text { Collision : } & f_{i}^{*}(\boldsymbol{x}, t)=f_{i}(\boldsymbol{x}, t)+\Omega_{i}(\boldsymbol{x}, t) \\
\text { Streaming : } & f_{i}\left(\boldsymbol{x}+\Delta t \boldsymbol{c}_{\boldsymbol{i}}, t+\Delta t\right)=f_{i}^{*}(\boldsymbol{x}, t)
\end{array}
$$

where $\Omega_{i}(x, t)$ symbolizes the collision operator and superscript $*$ refers to the post-collision quantities. The fluid density, $\rho$, and velocity vector field, $u=\left[u_{x}, u_{y}\right]$, are calculated by zeroth and first-order momentums of the population as expressed below: 


$$
\rho=\sum_{i} f_{i} ; \rho u=\sum_{i} f_{i} \boldsymbol{c}_{\boldsymbol{i}}
$$

The mean-field interaction force applies to simulate the molecular interactions, which lead to the phase separation. The pseudopotential (also known as effective mass) $\psi=\psi(\rho)$, is defined by the local density $(\rho)$. The summation of the pairwise interactions between particles at a given site $x$ and neghboring sites $x^{\prime}$ is considered as the interparticle force in the single-component multiphase (SCMP) LBM. Shan and Chen defined the lattice version of the interaction potential to introduce the long-range intermolecular interaction, as follows:

$$
V\left(x, x^{\prime}\right)=G\left(x, x^{\prime}\right) \psi(x) \psi\left(x^{\prime}\right)
$$

where $G\left(x, x^{\prime}\right)=G\left(x-x^{\prime}\right)$ is a Green's function. Based on the interaction potential, the interparticle forces $\left(\boldsymbol{F}_{\text {int }}\right)$ can be written as:

$$
\boldsymbol{F}_{\text {int }}(x, t)=-G\left|\boldsymbol{e}_{a}\right| \psi(x, t) \sum_{i=0}^{q} \psi\left(x+\boldsymbol{e}_{\boldsymbol{a}}, t\right) \boldsymbol{e}_{a}
$$

where $q$ symbolizes the number of the neighboring sites. The right-hand side of Equation (6) represents the finite difference form of $-\psi \Delta(\psi)$ and an unspecified relative interaction strength at different distances. The relative interaction strength is determined based on the finite difference gradient operator, which imposes the sufficient anisotropy to lower the spurious current velocity at a curved interface of multiphase. Following the standard analysis procedure of finite difference, the Taylor series of an arbitrary multi-dimensional function $f$ at lattice site $x$ and $[: a=1, \ldots, d]$ as a set of vector starting from $x$ to its $d$ neighbors is given below:

$$
f(x+e)=\Sigma_{\mathrm{n}=0}^{\infty} \frac{1}{n !}\left[\nabla^{(n)} f(x)\right] \cdot e e \cdots e
$$

where $x$ is a lattice site and the product of the right-hand side is a complete contraction between two rank-n tensor contractions. Based on the standard notation of Wolfram ${ }^{76}$, one can write: 


$$
E_{i_{1} i_{2} \ldots i_{n}}^{(n)}=\sum_{i} w\left(\left|\boldsymbol{e}_{a}\right|^{2}\right)\left(\boldsymbol{e}_{a}\right)_{i_{1}} \cdots\left(\boldsymbol{e}_{a}\right)_{i_{n}}
$$

in which, $w\left(\left|c_{i}\right|^{2}\right)$ are the weights. Considering the finite difference approximation to the gradient of $f$ in Equation (7) results in:

$$
\begin{aligned}
& \Sigma_{i} w\left(\left|\boldsymbol{e}_{a}\right|^{2}\right) f\left(x+\boldsymbol{e}_{a}\right) \boldsymbol{e}_{a}=(\nabla f) \cdot E^{(2)}+\frac{1}{3 !}\left(\nabla^{(3)} f\right) \cdot E^{(4)}+\frac{1}{5 !}\left(\nabla^{(5)} f\right) . \\
& E^{(6)}+\cdots
\end{aligned}
$$

Shan ${ }^{69}$ followed the above procedure and calculated weights, which leads to unit $E^{(2)}$ and isotropic

\begin{tabular}{|c|c|c|c|c|c|c|c|c|}
\hline & Tensor & $w(1)$ & $w(2)$ & $w(3)$ & $w(4)$ & $w(5)$ & $w(6)$ & $w(8)$ \\
\hline \multirow{3}{*}{$2 \mathrm{D}$} & $E^{(4)}$ & $1 / 3$ & $1 / 12$ & & & & & \\
\hline & $E^{(6)}$ & $4 / 15$ & $1 / 10$ & & $1 / 120$ & & & \\
\hline & $E^{(8)}$ & $4 / 21$ & $4 / 45$ & & $1 / 60$ & $2 / 315$ & & $1 / 5040$ \\
\hline \multirow{3}{*}{$3 \mathrm{D}$} & $E^{(4)}$ & $1 / 6$ & $1 / 12$ & & & & & \\
\hline & $E^{(6)}$ & $2 / 15$ & $1 / 15$ & $1 / 60$ & $1 / 120$ & & & \\
\hline & $E^{(8)}$ & $4 / 45$ & $1 / 21$ & $2 / 105$ & $5 / 504$ & $1 / 315$ & $1 / 630$ & $1 / 5040$ \\
\hline
\end{tabular}
$E^{(n)}$ (see Table 1).

Table 1: Calculated weights based on the unit $E^{(2)}$ and isotropic $E^{(n)}$ tensors in two and three dimensions.

By expanding the interaction forces (see Equation (6)) through the Taylor series, one can have the following expression ${ }^{66}$ :

$$
\boldsymbol{F}_{\text {int }}(x, t)=-G c^{2}\left[\psi \nabla \psi+\frac{1}{2} c^{2} \psi \nabla\left(\nabla^{2} \psi\right)+\cdots\right]
$$

The pressure tensor should be obtained to analyse the mechanical balance at the interface. As a general rule, the pressure tensor is given as follows ${ }^{77}$ :

$$
\Sigma P . A=\Sigma_{x} F
$$


in which, $P$ introduces the pressure and $A$ is the area. The analytical expression of the normal pressure tensor in the one-dimensional case is expressed as:

$$
P_{n}=c_{s}^{2} \rho+\frac{1}{2} G c^{2} \psi^{2}(\rho)+\frac{G c^{4}}{12}\left[\alpha\left(\frac{d \psi}{d n}\right)^{2}+\beta \psi \frac{d^{2} \psi}{d n^{2}}\right]
$$

in which, $\alpha=1-3 e_{4}, \beta=1+6 e_{4}$ where $e_{4}$ is the fourth-order tensor as given below ${ }^{77}$ :

$$
e_{4}=\frac{\omega_{1}}{2}+2 \omega_{2}+8 \omega_{4}+25 \omega_{5}+32 \omega_{8}+\cdots
$$

The bulk pressure $\left(p_{b}\right)$ is equal to first two diagonal terms of the pressure tensor in Equation (12). The pseudopotential can be defined based on the bulk pressure as ${ }^{70,78}$ :

$$
\psi(\rho)=\sqrt{\frac{2\left(P_{b}-c_{S}^{2} \rho\right)}{G c^{2}}}
$$

Different EOSs can be incorporated in the LBM through Equation (14).

Based on the BGK assumptions, the collision operator $\left(\Omega_{i}\right)$ is defined by a single relaxation of population with the same rate to a local equilibrium. The BGK collision operator is given below:

$$
\Omega_{i}=\frac{\left(f_{i}^{e q}-f_{i}\right)}{\tau}
$$

where $\tau$ denotes the single relaxation time scale, which is related to the kinematic viscosity of the fluid according to the following equation:

$$
v=\left(\tau-\frac{1}{2}\right) c_{s}^{2} \Delta t
$$

In Equation (15), $f_{i}^{e q}$ is the equilibrium PDF, as defined below: 


$$
f_{i}^{e q}=w_{i} \rho\left[1+3 \boldsymbol{c}_{\boldsymbol{i}} \cdot \boldsymbol{u}+\frac{9}{2}\left(\boldsymbol{c}_{\boldsymbol{i}} \cdot \boldsymbol{u}\right)^{2}-\frac{3}{2} \boldsymbol{u} \cdot \boldsymbol{u}\right]
$$

where $w_{i} s$ are a set of weights, which are determined based on the LB model.

In general, the collision process includes multiple physical quantities that may relax on the different time scales. To consider the information of the time scales, a full constant matrix $(\Lambda)$ is employed instead of a single time scale, $\tau$. Therefore, the collision operator can be rewritten as follows:

$$
\Omega_{i}=\Sigma_{j} \Lambda_{i j}\left(f_{j}^{e q}-f_{j}\right)
$$

Following the derivation presented by Premnath and Abraham ${ }^{79}$, by applying the transformation, $\overline{f_{i}}=f_{i}-1 / 2 \Delta t$, Equation (1) can be rewritten as follows:

$$
\begin{aligned}
\bar{f}_{i}\left(\boldsymbol{x}+\Delta t \boldsymbol{c}_{\boldsymbol{i}}, t\right. & +\Delta t) \\
& =\bar{f}_{i}(\boldsymbol{x}, t)+\left.\Sigma_{j} \Lambda_{i j}\left(f_{j}^{e q}-\bar{f}_{j}\right)\right|_{(x, t)}+\left.\Sigma_{j}\left(I_{i j}-\frac{1}{2} \Lambda_{i j}\right) S_{i}\right|_{(x, t)} \Delta t
\end{aligned}
$$

where $I_{i j}$ is the components of the identity matrix. Solving Equation (19) requires a complex matrix manipulation. A linear transformation can be used to diagonalize the collision matrix. The transformation matrix $\mathbf{T}$ transform the distribution functions from velocity space $\left(\bar{f}_{i}\right)$ into the moment space $\left(\hat{f}_{i}\right)$, which are related to the macroscopic physical quantities such as density, momentum, energy, and their flux. The transformation matrix form is related to the lattice structure. The explicit form of D2Q9 is presented below ${ }^{80}$ :

$$
\mathbf{T}=\left[\begin{array}{ccccccccc}
1 & 1 & 1 & 1 & 1 & 1 & 1 & 1 & 1 \\
-4 & -1 & -1 & -1 & -1 & 2 & 2 & 2 & 2 \\
4 & -2 & -2 & -2 & -2 & 1 & 1 & 1 & 1 \\
0 & 1 & 0 & -1 & 0 & 1 & -1 & -1 & 1 \\
0 & -2 & 0 & 2 & 0 & 1 & -1 & -1 & 1 \\
0 & 0 & 1 & 0 & -1 & 1 & 1 & -1 & -1 \\
0 & 0 & -2 & 0 & 2 & 1 & 1 & -1 & -1 \\
0 & 1 & -1 & 1 & -1 & 0 & 0 & 0 & 0 \\
0 & 0 & 0 & 0 & 0 & 1 & -1 & 1 & -1
\end{array}\right]
$$


The transformed form of Equation (19) into moment space can be calculated by multiplying the transformation matrix $\mathbf{T}$, as given below:

$$
\begin{aligned}
\hat{f}_{i}\left(\boldsymbol{x}+\Delta t \boldsymbol{c}_{\boldsymbol{i}}, t\right. & +\Delta t) \\
& =\hat{f}_{i}(\boldsymbol{x}, t)+\left.\Sigma_{\beta} \widehat{\Lambda}_{i \beta}\left(\hat{f}_{\beta}^{e q}-\hat{f}_{\beta}\right)\right|_{(x, t)}+\left.\Sigma_{\beta}\left(I_{i \beta}-\frac{1}{2} \widehat{\Lambda}_{i \beta}\right) \hat{S}_{i}\right|_{(x, t)} \Delta t
\end{aligned}
$$

The transformed collision matrix $\widehat{\Lambda}_{i \beta}=\mathrm{T} \Lambda \mathrm{T}^{-1}$ is become diagonal (for the D2Q9 lattice), as follows:

$$
\widehat{\Lambda}=\operatorname{diag}\left[s_{1}, s_{2}, s_{3}, s_{4}, s_{5}, s_{6}, s_{7}, s_{8}, s_{9}\right]
$$

The diagonal elements of $\widehat{\Lambda}, s_{1}$ through $s_{9}$, are the new relaxation parameters in associating with elements of $\hat{f}_{i}$.

According to Malaspinas ${ }^{81}$ and Coreixas ${ }^{82-83}$, the complete expression of the equilibrium distributions is

$$
\begin{aligned}
f_{i}^{e q}=w_{i} \rho[1 & +\frac{\boldsymbol{c}_{\boldsymbol{i}} \cdot \boldsymbol{u}}{c_{s}^{2}}+\frac{1}{2 c_{s}^{4}} \mathcal{H}_{i}^{(2)} \\
& : \boldsymbol{u} \boldsymbol{u}+\frac{1}{2 c_{s}^{6}}\left(\mathcal{H}_{i x x y}^{(3)} u_{x}^{2} u_{y}+\mathcal{H}_{\text {ixyy }}^{(3)} u_{x} u_{y}^{2}\right) \\
& \left.+\frac{1}{4 c_{s}^{8}} \mathcal{H}_{i x x y y}^{(4)} u_{x}^{2} u_{y}^{2}\right]
\end{aligned}
$$

with $\mathrm{w}_{0}=4 / 9, \mathrm{w}_{1 \ldots 4}=1 / 9, \mathrm{w}_{5 \ldots 8}=1 / 36, \mathrm{c}_{\mathrm{s}}=1 / \sqrt{3}$ is the lattice sound speed and $\mathcal{H}^{(\mathrm{n})}$ denotes the Hermite polynomial of order n. Notice that Eq.(23) degrades into the classical secondorder truncated formula if $\mathcal{H}^{(3)}$ and $\mathcal{H}^{(4)}$ are neglected.

In order to create a CMs-based collision operator, one should adopt lattice directions shifted by the local fluid velocity (see Geier et al. ${ }^{35}$ ), $\overline{\boldsymbol{c}}_{i}=\left[\left|\overline{\boldsymbol{c}}_{x i}\right\rangle,\left|\overline{\boldsymbol{c}}_{y i}\right\rangle\right]$, where

$$
\left|\overline{\boldsymbol{c}}_{x i}\right\rangle=\left|\boldsymbol{c}_{x i}-u_{x}\right\rangle
$$




$$
\left|\overline{\boldsymbol{c}}_{y i}\right\rangle=\left|\boldsymbol{c}_{y i}-u_{y}\right\rangle
$$

Then, we transform distributions into moments (and vice versa) by applying the matrix $\overline{\mathcal{T}}=$ $\left[\bar{T}_{0}, \ldots, \bar{T}_{i}, \ldots, \bar{T}_{8}\right]$ (see for example De Rosis and Luo ${ }^{86}$ ) where

$$
\begin{gathered}
\left|\bar{T}_{0}\right\rangle=|1, \ldots, 1\rangle \\
\left|\bar{T}_{1}\right\rangle=\left|\bar{c}_{x i}\right\rangle \\
\left|\bar{T}_{2}\right\rangle=\left|\bar{c}_{y i}\right\rangle \\
\left|\bar{T}_{3}\right\rangle=\left|\bar{c}_{x i}^{2}+\bar{c}_{y i}^{2}\right\rangle \\
\left|\bar{T}_{4}\right\rangle=\left|\bar{c}_{x i}^{2}-\bar{c}_{y i}^{2}\right\rangle \\
\left|\bar{T}_{5}\right\rangle=\left|\bar{c}_{x i} \bar{c}_{y i}\right\rangle \\
\left|\bar{T}_{6}\right\rangle=\left|\bar{c}_{x i}^{2} \bar{c}_{y i}\right\rangle \\
\left|\bar{T}_{7}\right\rangle=\left|\bar{c}_{x i} \bar{c}_{y i}^{2}\right\rangle \\
\left|\bar{T}_{8}\right\rangle=\left|\bar{c}_{x i}^{2} \bar{c}_{y i}^{2}\right\rangle
\end{gathered}
$$

We can evaluate pre-collision and equilibrium $\mathrm{CMs}$ as

$$
\begin{aligned}
\left|k_{i}\right\rangle & =\overline{\mathcal{T}}^{T}\left|f_{i}\right\rangle \\
\left|k_{i}^{e q}\right\rangle & =\overline{\mathcal{T}}^{T}\left|f_{i}^{e q}\right\rangle
\end{aligned}
$$

respectively, where

$$
\begin{gathered}
\left|k_{i}\right\rangle=\left[k_{0}, \ldots, k_{i}, \ldots, k_{8}\right]^{\top} \\
\left|k_{i}^{e q}\right\rangle=\left[k_{0}^{e q}, \ldots, k_{i}^{e q}, \ldots, k_{8}^{e q}\right]^{\top}
\end{gathered}
$$

and $\left|f_{i}^{e q}\right\rangle=\left[f_{0}^{e q}, \ldots, f_{i}^{e q}, \ldots, f_{8}^{e q}\right]^{\top}$. We obtain that only three equilibrium central moments are different from zero:

$$
\begin{gathered}
k_{0}^{e q}=\rho \\
k_{3}^{e q}=2 \rho c_{s}^{2} \\
k_{8}^{e q}=\rho c_{s}^{4}
\end{gathered}
$$


and $k_{1,2,4,5,6,7}^{e q}=0$. Indeed, as stated in De Rosis and Luo ${ }^{86}$, the discrete equilibrium CMs are equal to those of the continuous Maxwellian distribution when the full set of Hermite polynomials is considered. Let us denote the $9 * 9$ unit tensor as $\mathbf{I}$ and the $9 * 9$ relaxation matrix as $\Lambda=$ $\operatorname{diag}[1,1,1,1, \omega, \omega, 1,1,1]$. Following De Rosis et al. ${ }^{56}$, the post-collision state in terms of central moments is

$$
\left|k_{i}^{\star}\right\rangle=(\mathbf{I}-\Lambda) \overline{\mathcal{T}}^{\top}\left|f_{i}\right\rangle+\Lambda \overline{\mathcal{T}}^{\top}\left|f_{i}^{e q}\right\rangle+\left(\mathbf{I}-\frac{\Lambda}{2}\right) \overline{\mathcal{T}}^{\top}\left|\mathcal{F}_{i}\right\rangle
$$

The last ingredient to be defined is the forcing term $\mathcal{F}_{i}$. Similarly to the approach adopted for the equilibrium distribution (see Eq.(23)), Huang et al. ${ }^{84}$ suggested to expand the forcing term as

$$
\mathcal{F}_{i}=w_{i}\left(\frac{F}{c_{s}} \cdot \mathcal{H}^{(1)}+\frac{[F u]}{2 c_{S}^{2}} \cdot \mathcal{H}^{(2)}+\frac{[F u u]}{6 c_{s}^{3}} \cdot \mathcal{H}_{[x y y],[x x y]}^{(3)}+\frac{[F u u u]}{24 c_{s}^{4}} \cdot \mathcal{H}_{[x x y y]}^{(4)}\right)
$$

where the square bracket in Hermite coefficient denotes permutations. The popular formula by Guo et al. ${ }^{85}$ is then recovered when $\mathcal{H}^{(3)}$ and $\mathcal{H}^{(4)}$ are disregarded. The central moments of the discrete force term are ${ }^{56}$

$$
\left|R_{i}\right\rangle=\overline{\mathcal{T}}^{T}\left|\mathcal{F}_{i}\right\rangle
$$

where

$$
\begin{gathered}
\left|R_{1}\right\rangle=F_{x} \\
\left|R_{2}\right\rangle=F_{y} \\
\left|R_{6}\right\rangle=F_{y} c_{s}^{2} \\
\left|R_{7}\right\rangle=F_{x} c_{s}^{2}
\end{gathered}
$$

and $R_{0,3,4,5,8}=0$. These compact expressions are different from those by Huang et al. ${ }^{84}$ due to the choice of a different basis. Moreover, one can notice that Eqs.(32) can also be obtained when the velocity terms in Eqs(15) of De Rosis ${ }^{44}$ are neglected. Indeed, we remark the concept stated by De Rosis et al. ${ }^{56}$, where it has been demonstrated that the adoption of the Hermite polynomials of 
the maximum admissible order ( $\mathrm{n}=4$ in the D2Q9 space according to Malaspinas ${ }^{81}$ ) leads to Galilean-invariant (or, in other words, velocity-independent) equilibrium and forcing central moments.

This concise scheme leads to post-collision central moments that are (see De Rosis et al. ${ }^{56}$ ):

$$
\begin{gathered}
k_{0}^{\star}=\rho \\
k_{1}^{\star}=\frac{F_{x}}{2} \\
k_{2}^{\star}=\frac{F_{y}}{2} \\
k_{3}^{\star}=2 \rho c_{s}^{2} \\
k_{4}^{\star}=(1-\omega) k_{4} \\
k_{5}^{\star}=(1-\omega) k_{5} \\
k_{6}^{\star}=\frac{F_{y}}{6} \\
k_{7}^{\star}=\rho c_{s}^{4}
\end{gathered}
$$

Then, we can reconstruct post-collision populations $\left|f_{i}^{\star}\right\rangle=\left[f_{0}^{\star}, \ldots, f_{i}^{\star}, \ldots, f_{8}^{\star}\right]^{\top}$ by applying the backtransformation (see De Rosis ${ }^{55}$ )

$$
\left|f_{i}^{\star}\right\rangle=\left(\overline{\mathcal{T}}^{T}\right)^{-1}\left|k_{i}^{\star}\right\rangle
$$

Incorporating equations of state. In this part, Peng-Robinson (PR) EOS, as one of most successive EOSs in both academic and industrial sectors ${ }^{87}$, and cubic plus association (CPA) EOS ${ }^{73}$, as an accurate and computational cost effective EOS in simulating associating fluids, Shen and Chen EOS (SC EOS), an original EOS which implemented in pseudopotential LBM, and Sbragaglia and Shan EOS (SS EOS), a thermodynamic consistence EOS, are briefly reviewed.

PR as a cubic EOS was developed due to the low accuracy of Soave-Redlich-Kwong (SRK) EOS 72 in predicting the liquid density of heavy hydrocarbons. The liquid density calculated by SRK is usually higher than the experimental measured values ${ }^{88}$. Peng and Robinson EOS followed the same strategy as SRK model in thermodynamic modeling, except the van der Waals attractive term was modified as given below: 


$$
P=\frac{R T}{v-b}-\frac{a}{v(v+b)+b(v-b)}
$$

in which

$$
\begin{gathered}
a=a_{c} \alpha(T) \\
\alpha=\left[1+\left(0.37464+1.54226 \omega-0.26992 \omega^{2}\right)\left(1-\sqrt{T_{r}}\right)\right]^{2} \\
a_{c}=0.45724 \frac{R^{2} T_{c}^{2}}{P_{c}}, b=0.07780 \frac{R T_{c}}{P_{c}}
\end{gathered}
$$

where $P$ stands for the pressure; $R$ denotes the universal gas constant; $v$ introduces the molar volume; $a$ and $b$ are the attraction and repulsion terms, respectively; $\alpha$ represents the temperature dependence function in the attraction term; and the subscript $c$ refers to the critical properties. Second term in Equation (35) is the attraction term. Peng and Robinson added $b(v-b)$ to the attraction term, which improves the attractive pressure force impact.

CPA EOS was proposed based on a cubic EOS and the statistical associating fluid theory (SAFT) for modeling of physical interactions and associating forces, respectively. The CPA EOS reduces to a cubic EOS in the absence of associating force (e.g., hydrogen bonding compounds). Beside the simplicity and accuracy, the numerical implementation of the association term guarantees the computational time effectiveness of the CPA EOS. SRK EOS was used to describe the physical part in the original form introduced by Kontogeorgis et al. ${ }^{75}$. Firoozabadi proposed to use PR EOS as the physical part of CPA EOS to be suitable for thermodynamic modeling of hydrocarbon reservoir fluids ${ }^{89}$. The association term consists of the specific site-site interactions due to hydrogen bonding. The association interactions are listed in two categories, namely; selfassociation and cross-association for the same and different species, respectively. The bulk pressure of the system represented by CPA EOS is as follows:

$$
\begin{gathered}
P=\frac{R T}{v-b}-\frac{a}{v(v+b)+b(v-b)}-\frac{1}{2} \frac{R T}{v}\left(1-v \frac{\partial \ln g}{\partial v}\right) \Sigma_{i} x_{i} \Sigma_{A_{i}}\left(1-X_{A i}\right), i \\
=1,2, \ldots, n_{c}
\end{gathered}
$$


in which, $x$ symbolizes the mole fraction; $g$ introduces the hard sphere radial distribution function (RDF); $A_{i}$ refers to the active association site; and $X_{A i}$ stands for the fraction of site $A$ on component $i$ that does not form association with other sites, as presented below:

$$
\begin{gathered}
\mathrm{g} \approx \frac{2-\eta}{2(1-\eta)^{3}} \quad \text { where } \eta=\frac{b}{4 v} \\
\mathrm{X}_{\mathrm{Ai}}=\frac{1}{1+\sum_{j=1}^{n_{c}} \sum_{k=A, B, \ldots} \rho x_{j} X_{k j} \Delta_{A i}^{k j}}
\end{gathered}
$$

where $\rho=1 / v$ is the density and $\Delta_{A i}^{k j}$ introduces the self-association strength between sites $A$ and $B$, as defined below:

$$
\begin{gathered}
\Delta_{A i}^{k j}=g\left[\exp \left(\frac{\epsilon^{A_{i} K_{i}}}{R T}\right)-1\right] b_{i j} \beta^{A_{i} K_{i}} \\
\mathrm{~b}_{\mathrm{ij}}=\frac{b_{i}+b_{j}}{2}
\end{gathered}
$$

in which, $\epsilon^{A_{i} K_{i}}$ and $\beta^{A_{i} K_{i}}$ refer to the self-association energy and bonding volume parameters, respectively. Like the study conducted by Asadi and Zendehboudi ${ }^{74}$, we use four-site scheme (4C) and two site model (2B) for water ${ }^{89}$ and alcohols ${ }^{90}$, respectively. To keep consistency with the literature, the attraction, repulsion parameters, and universal gas constant of CPA EOS are supposed to be $a=2 / 49, b=2 / 21$, and $R=1$, respectively, in the lattice units ${ }^{70,91}$.

The original EOS proposed by Shan and Chen is determined by defining the effective mass as follows:

$$
\psi(\rho)=\rho_{0}\left[1-e^{-\frac{\rho}{\rho_{0}}}\right]
$$

where $\rho_{0}$ is a constant. Based on Eq. 14, the SC EOS can be obtained as:

$$
P=\frac{\rho}{3}+\frac{c_{0}}{2} G \rho_{0}^{2}\left[1-e^{-\frac{\rho}{\rho_{0}}}\right]^{2}
$$


According to the critical characteristic condition of single component fluids (i.e., $\left(\frac{\partial P}{\partial v}\right)_{T}=$ $\left.\left(\frac{\partial^{2} P}{\partial v^{2}}\right)_{T}=0\right)$, the critical properties of SC EOS are calculated as follows:

$$
\begin{gathered}
\rho_{c}=\rho_{0} \ln 2, \\
G_{c}=-\frac{4}{3 \rho_{o}} \\
T_{c}=-\frac{1}{g_{c}}=\frac{3}{4} \rho_{0}, \\
P_{c}=\rho_{0}\left(\ln 2-\frac{1}{6}\right)
\end{gathered}
$$

Therefore, two-phase separation of the system is guaranteed when $G<G_{c}$ and $\rho_{v}<\bar{\rho}<\rho_{l}$ where $\rho_{v}, \bar{\rho}$, and $\rho_{l}$ are the gas phase, average, and liquid densities, respectively. When $G<G_{c}$, the temperature of the system is lower than critical temperature which means two phases might exist and when the average pressure of the system is between two phase density means that two phases should be exists.

Sbragaglia and Shan introduced a new consistence pseudo-potential as follows ${ }^{65}$ :

$$
\psi(\rho)=\left(\frac{\rho}{\epsilon+\rho}\right)^{1 / \epsilon}
$$

where $\epsilon=-2 \alpha / \beta$. The critical properties of SS EOS are obtained by the following equations:

$$
\begin{gathered}
\rho_{c}=-\frac{\epsilon-2}{2}, G_{c}=\frac{1}{12}\left(\epsilon^{2}-4\right)\left(\frac{2-\epsilon}{2-\epsilon}\right)^{-\frac{2}{\epsilon}}, T_{c}=-\frac{1}{g_{c}} \\
=\frac{12}{\left(4-\epsilon^{2}\right)\left(\frac{2-\epsilon}{2-\epsilon}\right)^{-\frac{2}{\epsilon}}, P_{c}=\frac{(\epsilon-2)^{2}}{24}}
\end{gathered}
$$

Customized EOS. The numerical instability happens within the narrow regions of the interface, which fall in the unstable part of the EOS with $\left(\frac{\partial p}{\partial v}\right)_{T}>0$. Due to unphysical meaning of the 
unstable branch, it can be modified. By increasing the slope of the unstable branch, the internal forces are increased toward the liquid side of spinodal point while the internal forces are lowered toward the vapor region ${ }^{92}$. Colosqui et al. proposed a piecewise linear EOS in the unstable branch which is discontinuous in its first derivation ${ }^{92}$. Asadi and Zendehboudi introduced a new perturbation in the unstable branch that increases the slope and is continuous in its first derivation at the same time as given below ${ }^{74}$ :

$$
P_{\text {Customized }}=P_{C P A}+\delta P
$$

where,

$$
\delta P=\left\{\begin{array}{cc}
0 & \text { if } v<v_{l} \\
\kappa\left(\sin \left(\frac{\pi\left(v-v_{l}\right)}{L_{1}}+\frac{3 \pi}{2}\right)+1\right) & \text { if } v_{l}<v<v_{M} \\
\kappa\left(\sin \left(\frac{\pi\left(v-v_{l}\right)}{L_{2}}+\frac{\pi}{2}\right)+1\right) & \text { if } v_{M}<v<v_{g} \\
0 & \text { if } v_{g}<v
\end{array}\right.
$$

in which, $v_{m}=v_{g}-\alpha\left(v_{g}-v_{l}\right) . v_{g}$ and $v_{l}$ stand for the spinodal molar volumes on the gas and liquid sides, respectively. The perturbation is determined by a pair of $(\alpha, \kappa)$, individually.

\section{RESULTS AND DISCUSSION}

In the current research work, the central-based collision operator is implemented in the pseudopotential LBM. The flexibility of common pseudopotentials to predict the thermodynamic properties of a fluid is evaluated. The capability of implemented collision operator is determined by comparing the stability of central-moments-based LBM and MRT collision operator in the double layer shear test. A static drop test is selected to show the impact of isotropy order of gradient operator on the stability of the approach. The spurious velocity of static drop case is also studied. The thermodynamic consistency is examined by comparing the two-phase densities simulated by the proposed approach and calculated by Maxwell equal area construction. Finally, the Laplace law test is performed by determining the pressure difference of two phases at the different radius of the droplet.

Thermodynamically behavior of EOSs. Before providing further details of the central-based LBM, we briefly compare the thermodynamic behaviors simulated by different EOSs. Although 
SC and SS are mainly used in LBM studies, EOSs such as PR and CPA are more suitable to determine the fluid properties. Figure 1 shows the algebraic form of cubic equations, schematically. There is no physical meaning for branches with $v<b$. For cases with $p<p_{1}$, there are three roots $-(\sqrt{2}+1) b<v<0,0<v<(\sqrt{2}-1) b$, and $v>b$. The root with $v>b$ is physically acceptable and considered as the liquid phase density. For $p_{2}<p<p_{1}$, there is only one root, which belongs to the liquid phase density. When $p_{3}<p<p_{2}$, three roots are found. The highest belongs to gas phase, the lowest one corresponds to liquid phase, and the intermediate one has no physical meaning. In some cases, the negative pressure determines the liquid phase density (see panel $b$ of Figure 1$)^{89,93}$. CPA EOS exhibits a behavior similar to cubic EOSs at the acceptable branches.

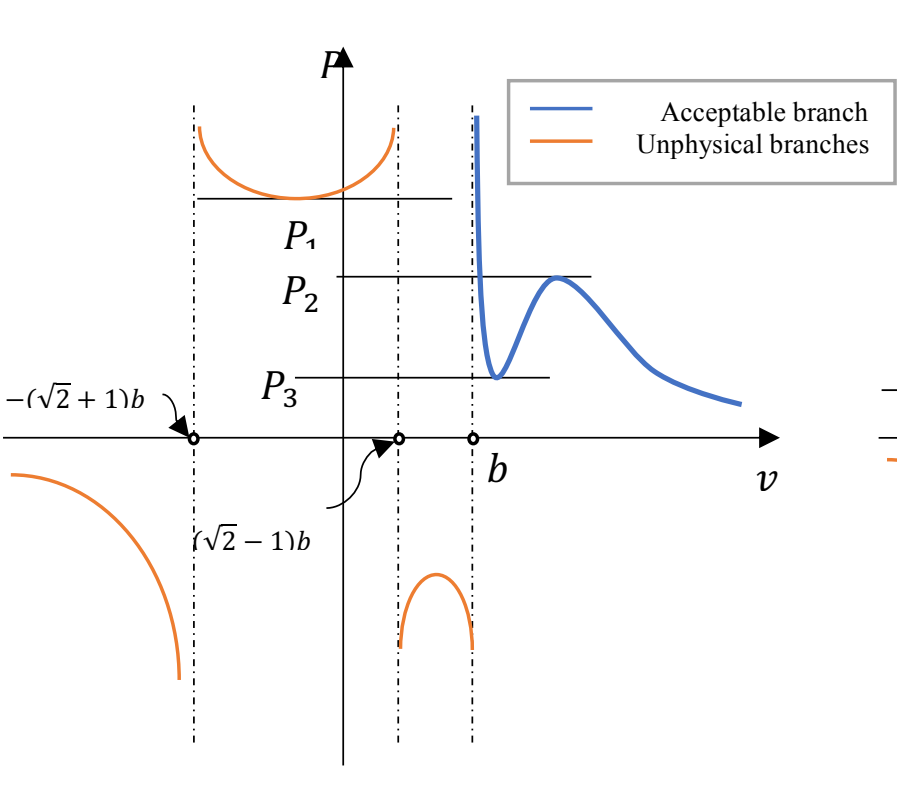

(a)

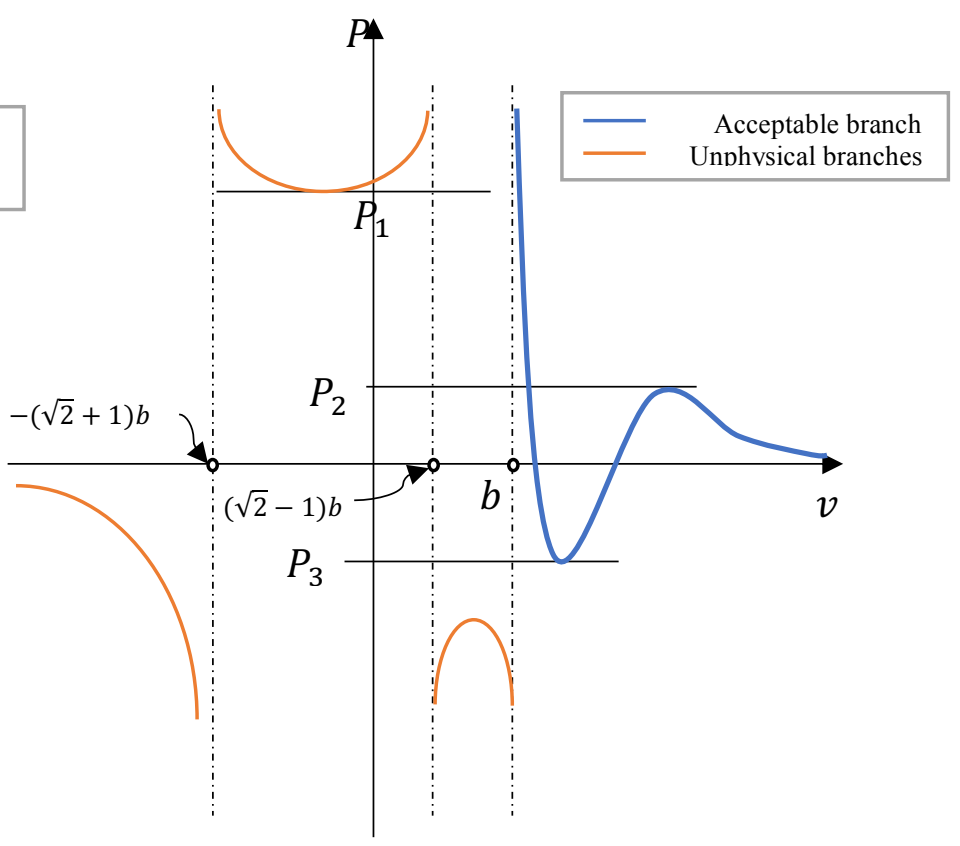

(b)

Figure 1: Algebraic behavior and various branches of cubic and CPA EOSs: a) without negative pressure possibility, and b) with negative pressure.

Figure 2 illustrates the algebraic form of LBM based EOS (e.g., SS and SC EOSs) for $T<T_{c}$. Both EOSs have a vertical asymptote at $v=0$, implying that the volume of particles is neglected. Both EOSs are not considerably affected upon changing their parameters, keeping their low 
flexibility/potential in predicting thermodynamic properties of fluids. Additionally, the negative pressure is not calculated by LBM based EOS.

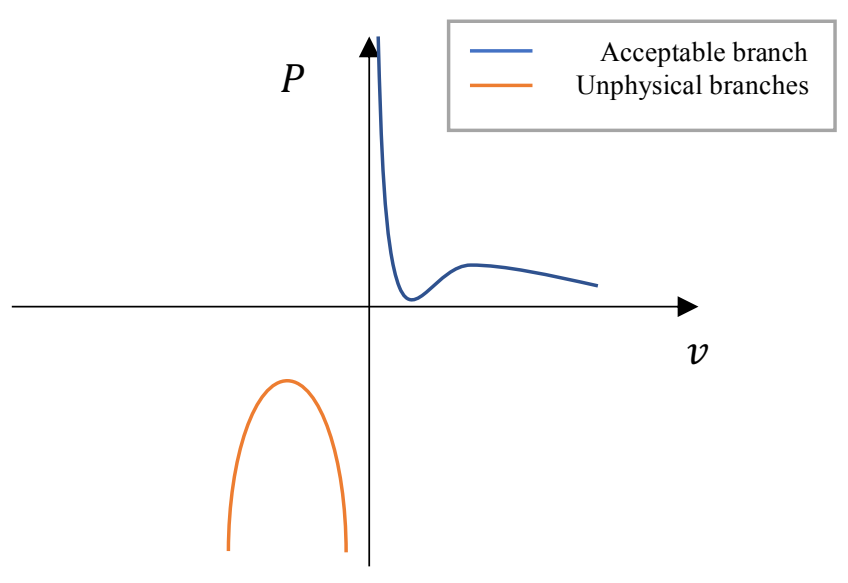

(a)

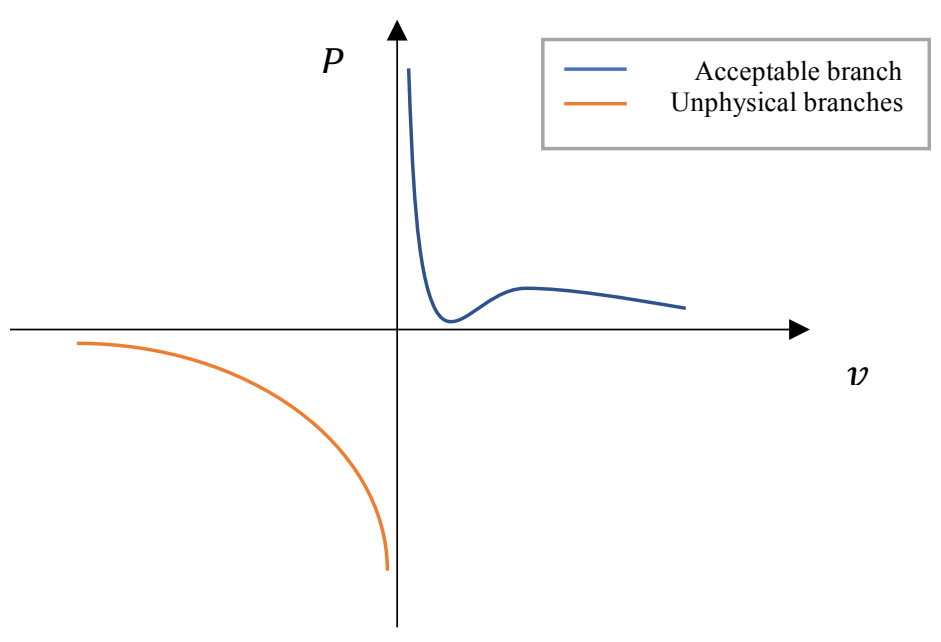

(b)

Figure 2: Various branches of a) SS and b) SC EOSs.

More importantly, the LBM based EOS has only one parameter that can be used to fit the experimental data. Figure 3 presents the calculated phases density at the equilibrium condition based on the Maxwell equal area construction. According to Figure 3, the SS EOS proposed by Sbragaglia and Shan has a low thermodynamic consistency with the experimental data and $\epsilon$ parameter has a low influence on the reduced properties; however, a good consistency with LBM simulation results is reported. Beside the inconsistency issue of SC EOS, it leads to the same reduced properties at different magnitudes of $v_{0}$ (see Equation (12)). In the rest of this study, the SS and SC EOSs are not discussed for evaluation purpose. 


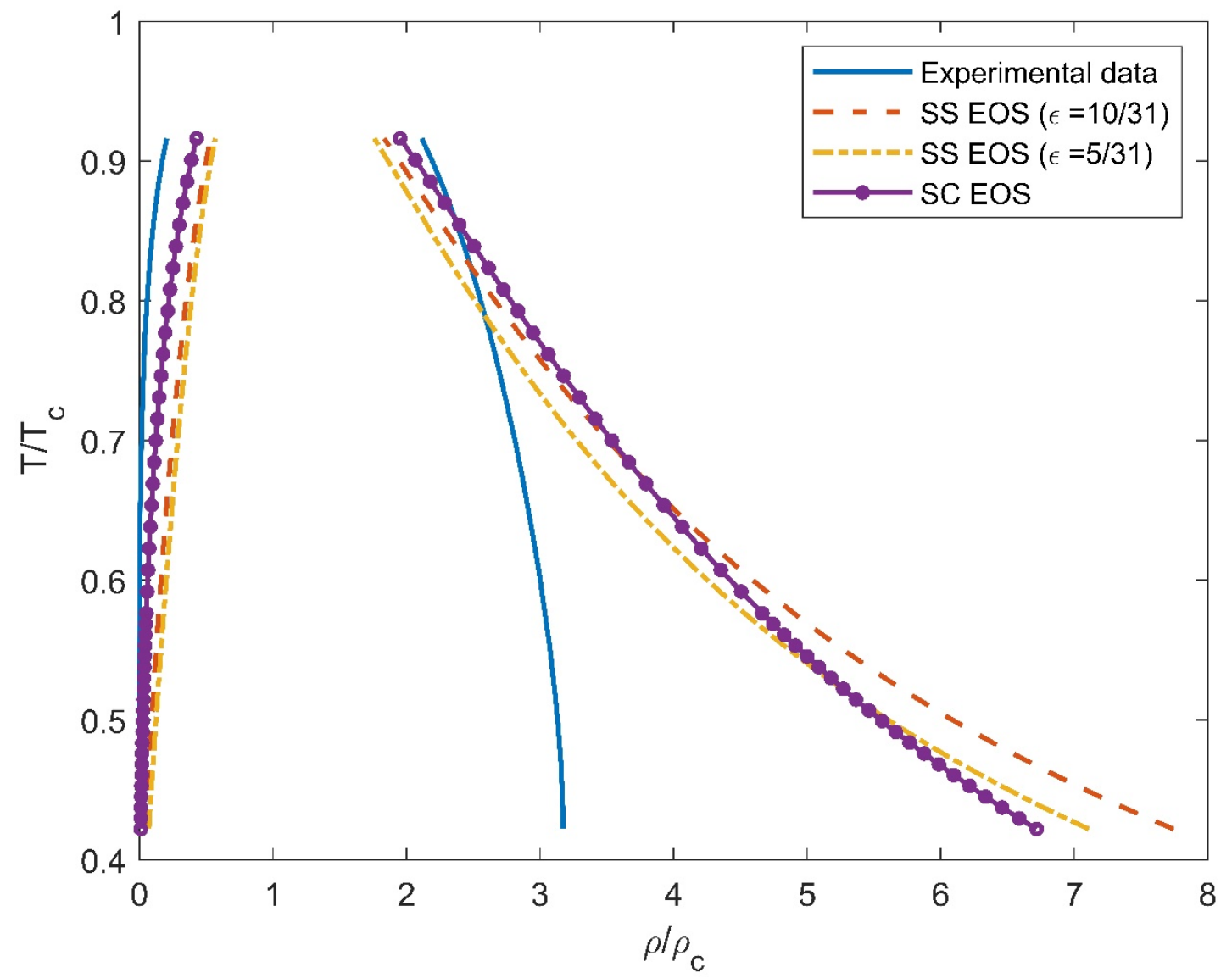

Figure 3: Comparison between calculated reduced density of two phases at equilibrium by EOS and experimental date.

Double shear layer test. To better demonstrate the dynamic properties of introduced CMs LBM, the results calculated by MRT and CMs collision operators were compared in the double shear layer test ${ }^{94}$, which is known as an excellent candidate to examine the numerical scheme's stability 82, 95-96. A fully periodic $2 \mathrm{D}$ boundary condition with $(x, y) \in[0, L]^{2}$ is considered. The initial flow filed consists of two longitudinal shear layers and transvers perturbation, as superimposed below:

$$
u_{x}= \begin{cases}u_{0} \tanh \left(\kappa\left(\frac{y}{L}-\frac{1}{4}\right)\right), & \frac{y}{L} \leq \frac{1}{2} \\ u_{0} \tanh \left(\kappa\left(\frac{3}{4}-\frac{y}{L}\right)\right), & \frac{y}{L}>\frac{1}{2}\end{cases}
$$

and 


$$
u_{y}=u_{0} \delta \sin \left(2 \pi\left(\frac{x}{L}+\frac{1}{4}\right)\right)
$$

where $\kappa=80$ and $\delta=0.05$. Fluid dynamics predicts the sheer layers and the generation of two controlling vortices based on the Kelvin-Helmotz instability mechanism. The Reynolds number is $R e=u_{0} L / v=3 \times 10^{4}$ and Mach number is assumed to be $M a=u_{0} / c_{s}=0.57$. The simulation is performed when $L=256$ and sixth-order Hermite polynomial is implemented for central moments based LBM. Figure 4 duplicates the mean kinetic energy (normalized by $u_{0}$ ) versus time for both central moments-based LBM and MRT. In the sixth-order Hermit polynomial case of central moments-based LBM, its performance is stable while it becomes unstable at early stages in the MRT case.

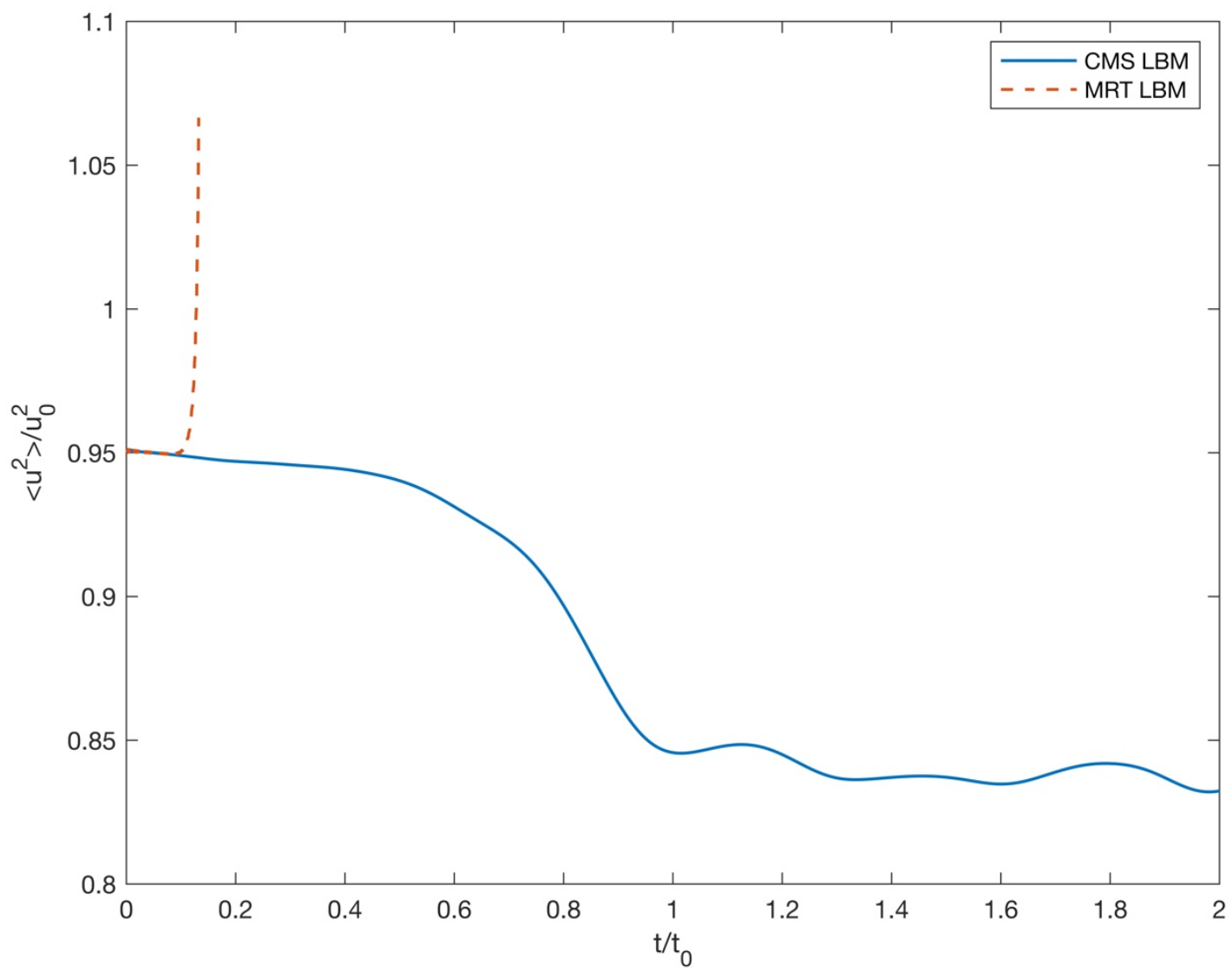

Figure 4: Double shear layer: comparison of time evolution of the normalized mean energy at $M a=0.57$ when the central moments-based LBM (solid line) and MRT (dash line) are implemented. 
Spurious velocity. To investigate spurious velocity, a static single-component bubble is placed in a 2D computational domain. A $100 \times 100$ lattice computational domain with a periodic boundary condition in all directions are considered in all tests. Initially, a droplet with a radius of $R_{0}=30$ is placed in the center of the domain with the vapor (liquid) phase outside (inside) the droplet. The initial phases density is selected based on the equilibrium phase density calculated by Maxwell construction and its initial distribution, as determined below:

$$
\begin{aligned}
& \rho(x, y)=\frac{\rho_{\text {liquid }}+\rho_{\text {gas }}}{2} \\
& \quad-\frac{\rho_{\text {liquid }}-\rho_{\text {gas }}}{2}\left[\tanh \left(\frac{2 \sqrt{\left(x-x_{c}\right)^{2}-\left(y-y_{c}\right)^{2}}-R_{0}}{w}\right)\right]
\end{aligned}
$$

where $w$ refers to the initial interface thickness; $x_{c}$ and $y_{c}$ are the droplet center position; and $R_{0}$ is the initial droplet radius. Following the approach by Asadi and Zendehboudi ${ }^{74}$, a new stability condition is applied. PR and CPA EOSs' parameters are selected as $R=1, a=2 / 49, b=2 / 21$, $\omega=0.344, \epsilon^{A B}=0.1636$, and $\beta=0.0973$.

The spurious velocity, also known as parasite current, is referred to the unphysical circular velocities near multiphase interface, which appears in the numerical simulations such as Level-set, Volume of fluid (VOF), and LBM ${ }^{97}$. The spurious velocity reduces the accuracy of the simulation and cannot be distinguished from the actual velocity, leading to instability problems in severe cases. The spurious velocity and surface tension are related to each other. High spurious velocity is one of the major drawbacks of the pseudopotential LBM, compared to the other LBM approaches such as free-energy LBM method. In general, higher spurious velocities are observed at high density ratios (lower temperature for single-component simulation cases) and low viscosities ${ }^{33,70}$.

In spite of MRT, central-moment-based LBM does not involve freely adjustable relaxation times; therefore there is no need to tune the free parameters. Figure 5 shows the spurious velocity at the steady-state condition calculated by the central-moment-based LBM. A dramatic decrease in the maximum spurious velocity is notable (up to 34 percent) by increasing the anisotropy order of the gradient operator, which indicates the enhancement in the stability method. In the higher anisotropy of gradient operators such as $8^{\text {th }}$ order of gradient operator, more neighbour lattice 
nodes are considered in the simulation. Hence, a wider interfacial thickness (e.g., lower interfacial tension) may cause some instabilities, particularly in low kinematic viscosities. To increase the interfacial tension, the attraction parameter $(a)$ is increased. Following the procedure introduced by Asadi and Zendehboudi ${ }^{74}$, the association parameters are updated for the new case, which are $\omega=0.344, \epsilon^{A B}=0.3410$, and $\beta=0.0887$ as the acentric factor and new association parameters in the CPA EOS for water. The reduced temperature is kept constant for all simulation runs.

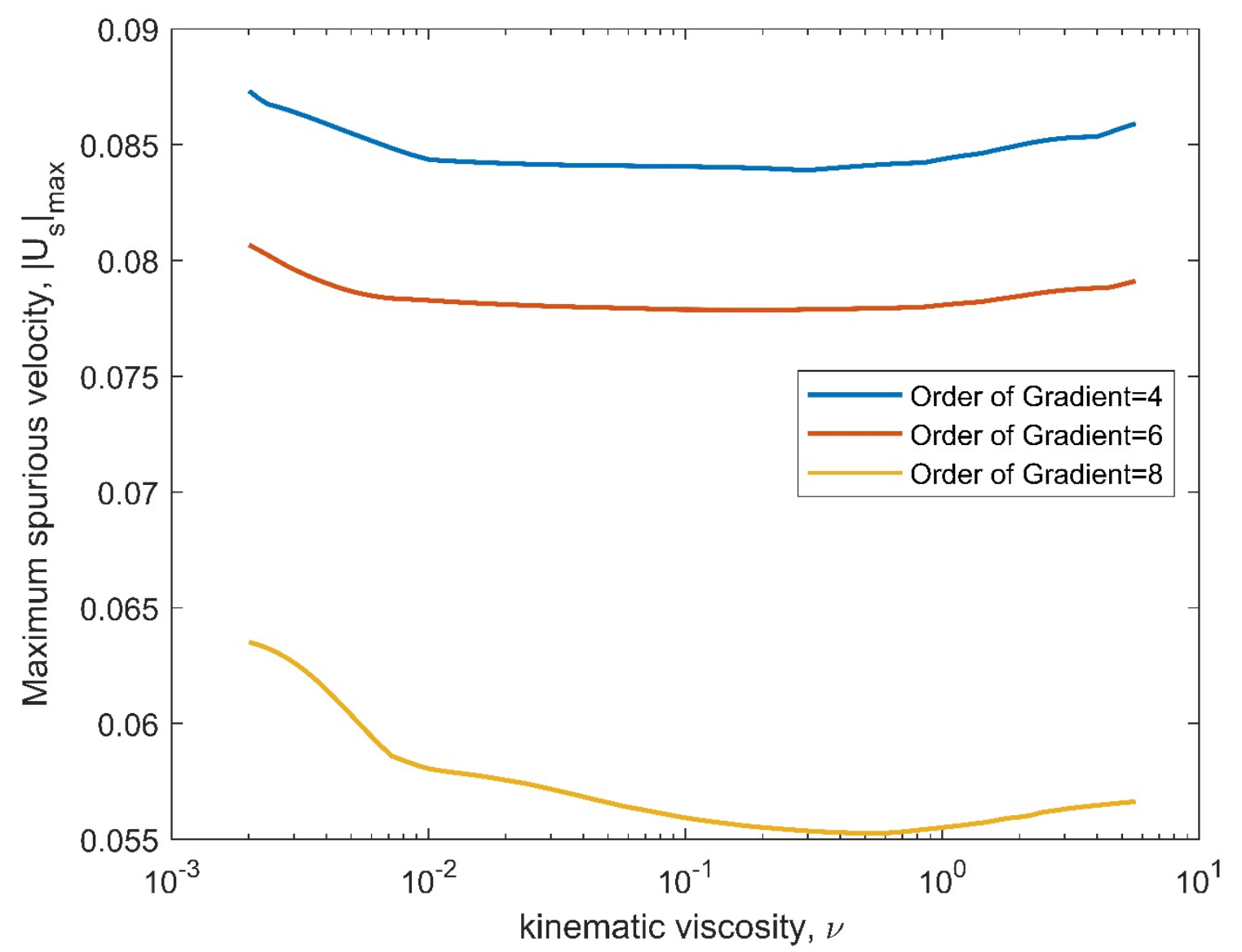

Figure 5: Maximum spurious velocity $\left|U_{s}\right|_{\text {max }}$ at the steady state condition versus kinematic viscosity of fluid $(v)$. For $4^{\text {th }}$ and $6^{\text {th }}$ order of gradient operator, the maximum spurious velocity is calculated at $a=$ $2 / 49, b=2 / 21, R=1$, and $T_{r}=0.9$. For $8^{\text {th }}$ case, the attractive term in the EOS is changed to $a=4 / 49$. 
(a)

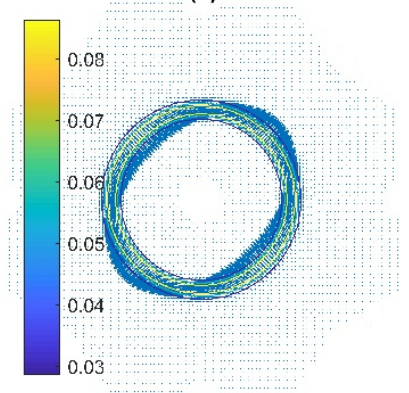

(b)

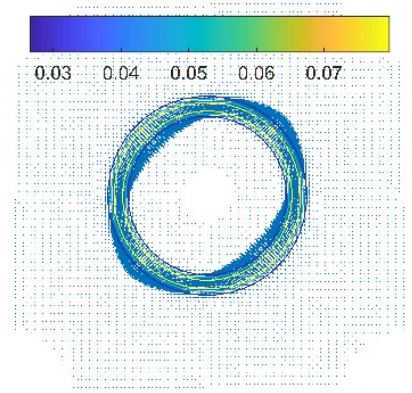

(c)

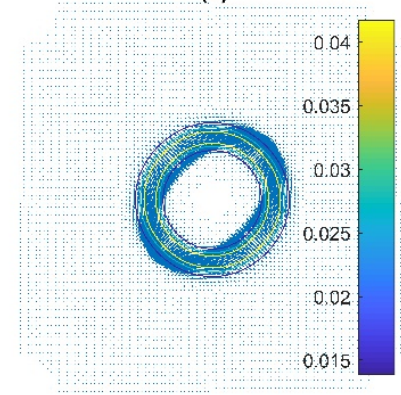

Figure 6: Velocity field and contour for a static droplet on the basis of different anisotropy orders of gradient operator: a) $4^{\text {th }}$, b) $6^{\text {th }}$, and c) $8^{\text {th }}$. CPA EOS is implemented in the central-moments-based LBM when $a=4 / 49, b=2 / 21, R=1, T_{r}=0.9$, and $\vartheta=1 / 6$.

Figure 6 duplicates the velocity field for central-moment-based Lattice Boltzmann at different anisotropy orders of gradient operators. For all cases, the thermodynamic parameters are the same and calculated at the same reduce temperature and kinematic viscosity $(\vartheta=1 / 6)$. A decrease in the magnitude of spurious velocity occurs with increasing the anisotropy order of gradient operator. In addition, the spurious velocity contours are changed, implying alteration in the interfacial thickness; the size of the droplet is also altered. For all three cases, the initial density is same and selected based on the Maxwell constriction; however, the thermodynamic consistency of the LBM results will change by changing the anisotropy order of gradient operator. Therefore, the size of droplet will be changed to maintain equilibrium condition. The thermodynamic consistency will be further discussed in this section. 


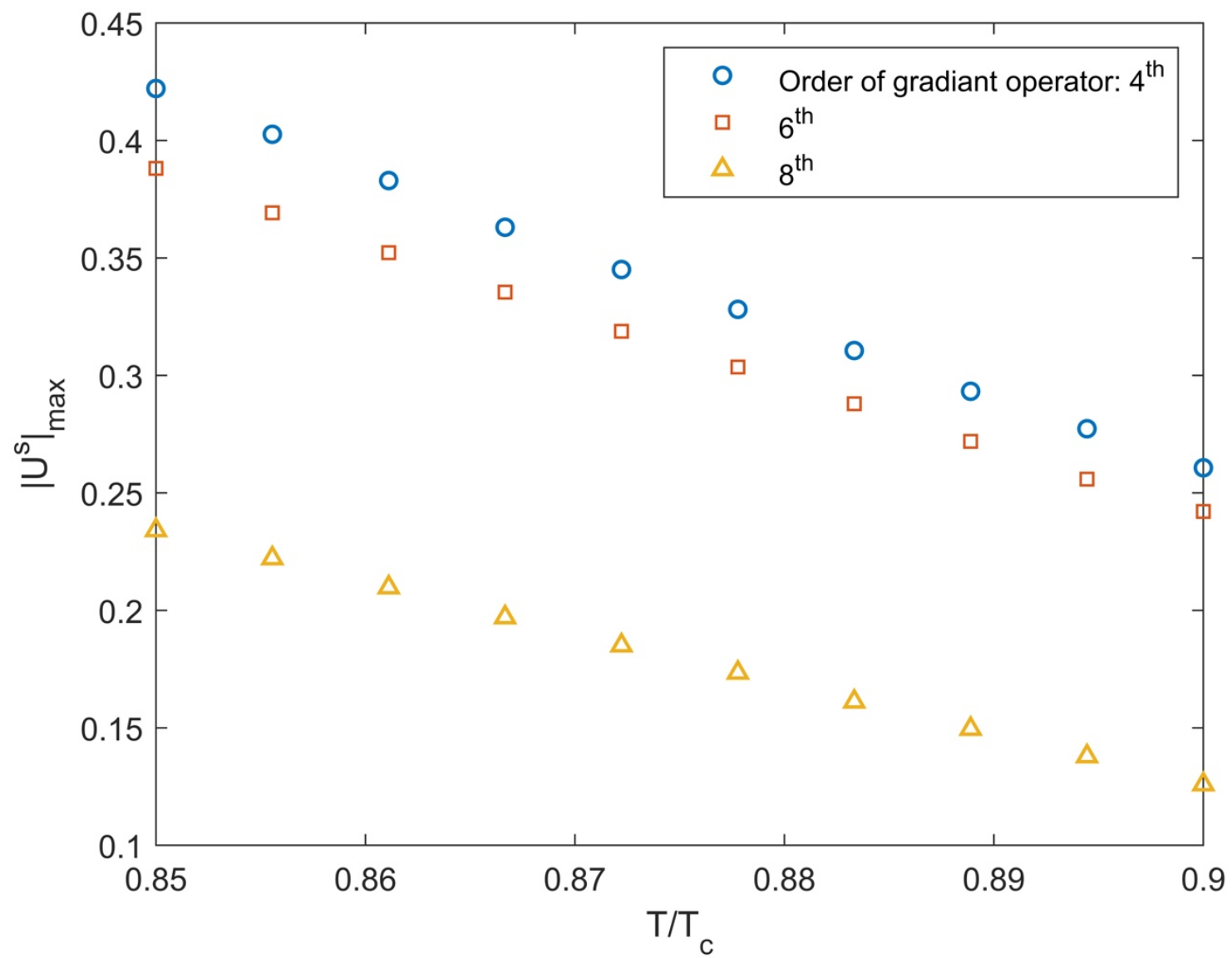

Figure 7: Impact of anisotropy order of gradient operator on the maximum spurious velocity at the steady state condition.

Figure 7 presents the influence of the anisotropy order of gradient operator on the maximum magnitude of spurious velocity at different reduced temperatures. As a general trend for all these three cases, the spurious velocity will increase as the reduced temperature is decreased. A decline in the reduced temperature leads to an increase in the density ratio (even by considering the thermodynamic inconsistency of the model) and consequently an increase in the spurious velocity.

Thermodynamic consistency. Sbragaglia and Shan ${ }^{65}$ showed that the consistency of the pseudopotential LBM is related to $\epsilon$. Shan ${ }^{77}$ also concluded that $\epsilon$ is a function of anisotropy order of gradient operator (see Equation (13)); therefore, the anisotropy order of gradient operator affects the thermodynamic consistency. Figure 8 illustrates the thermodynamic consistency at various density ratio conditions while using different orders of gradient operator. The thermodynamic input parameters for the EOS are kept the same as the case utilized in Figure 5. 
As it is shown in Figure 6, there is a smaller liquid drop for the case with $8^{\text {th }}$ order of gradient operator while all initial parameters were same for all cases; therefore, the density of the liquid phase is decreased, while the gas density is increased. In addition, the equilibrium gas density in the $8^{\text {th }}$ order of gradient operator is higher than that calculated by Maxwell equal area construction. It has also a lower density for the liquid phase. To approach the equilibrium condition, the liquid phase density should be then lowered and the gas phase density should be increased when the initial densities of both phases are selected based on the Maxwell equal-area construction. This eventually causes a decrease in the droplet radius (see Figure 6). It should be mentioned that the liquid phase pressure in some cases will become negative that has a physical meaning (see Figure 1 and Refs. ${ }^{89,}{ }^{93}$ ) when the attraction parameter is fixed at $a=4 / 49$. The thermodynamic inconsistency is notable; however, $\mathrm{Li}$ et al. suggested a method to improve the thermodynamic consistency by improving the forcing scheme ${ }^{66}$.

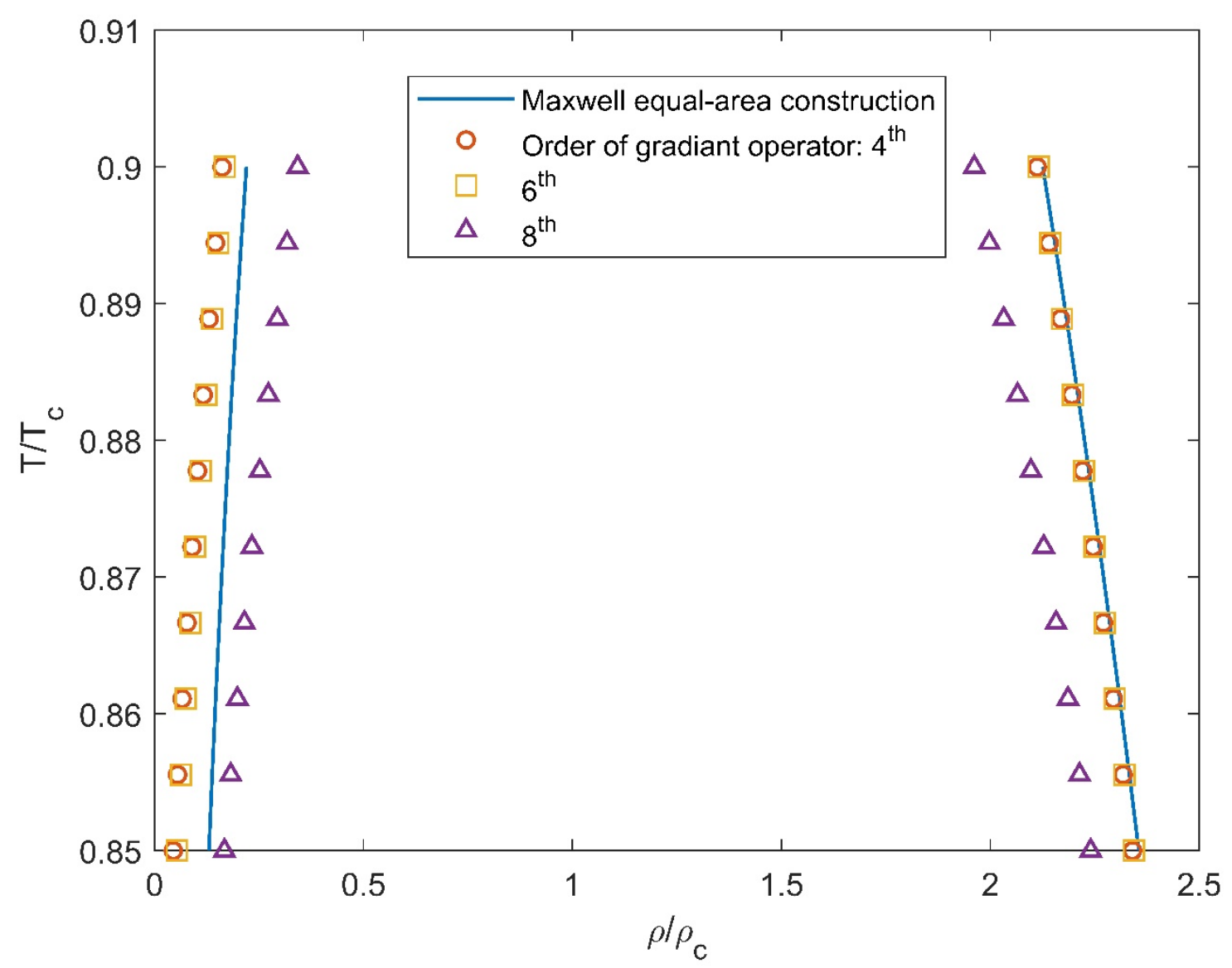

Figure 8: Thermodynamic consistency of the central-moments-based LBM at different anisotropy orders of gradient operator. 
A higher anisotropy order of gradient operator makes possible simulation of a multiphase fluid with a higher density ratio (lower reduced temperatures for single component multiphase cases), while employing the pseudopotential central-moments-based LBM. As the reduced temperature is decreased, the interfacial tension will be increased. Hence, there is no need to increase the interfacial tension by increasing the attraction term in the CPA EOS. Despite the mid-range approach, there is no direct parameter to tune the interfacial tension/thickness ${ }^{68,98-99}$, while tuning the EOS input parameters is a strategy to tune the interfacial tension/thickness. However, changing the EOS input parameters may affect the thermodynamic consistency.

Laplace law test. Laplace law test is performed by placing a static droplet in the fully periodic domain. The initial densities are selected based on the Maxwell equal-area construction. After reaching the defined steady-state condition, the pressure can be calculated by EOS. Figure 9 duplicates a scaled image of density at the steady-state condition. As stated by Mukherjee et al., the exact location of droplet edge is uncertain ${ }^{100}$. Therefore, a subpixel edge location based on partial area effect that is an image processing technique is implemented to increase the accuracy of droplet radius determination ${ }^{101}$. The droplet edge is determined by the red circles in Figure 9. The inner plot shows the pressure trend along the cross section (red line). The horizontal parts determine the pressure of bulk phases. The difference between two phases pressure is related to Laplace pressure. The pressure trend between two phases is because of the unstable part of the EOS with $\left(\frac{\partial \mathrm{p}}{\partial \mathrm{v}}\right)_{\mathrm{T}}>0$. The pressure difference versus the inverse of droplet radius is presented in Figure 10. According to Figure 10, the pressure difference increases with lowering the radius. All cases are simulated when $a=2 / 49, b=2 / 21$, and $T_{r}=0.9$. A linear relationship between the pressure difference $\Delta p$ and inverse of droplet radius is observed as given by Laplace law below:

$$
\Delta p=\frac{\sigma}{R}
$$




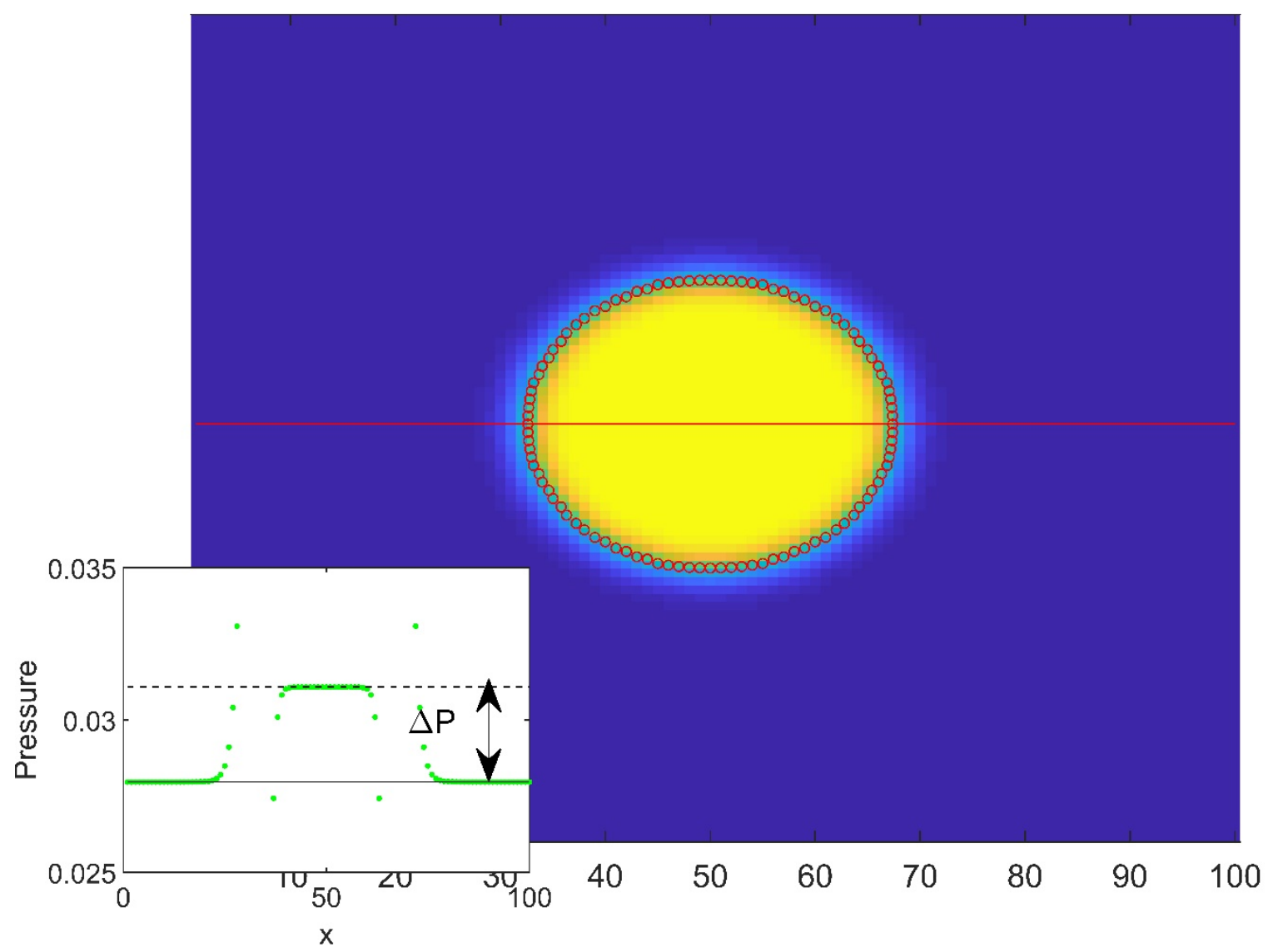

Figure 9: Density image and pressure variation along a cross line. Red circles are the droplet edge. Inset plot depicts the pressure distribution along the red line. 


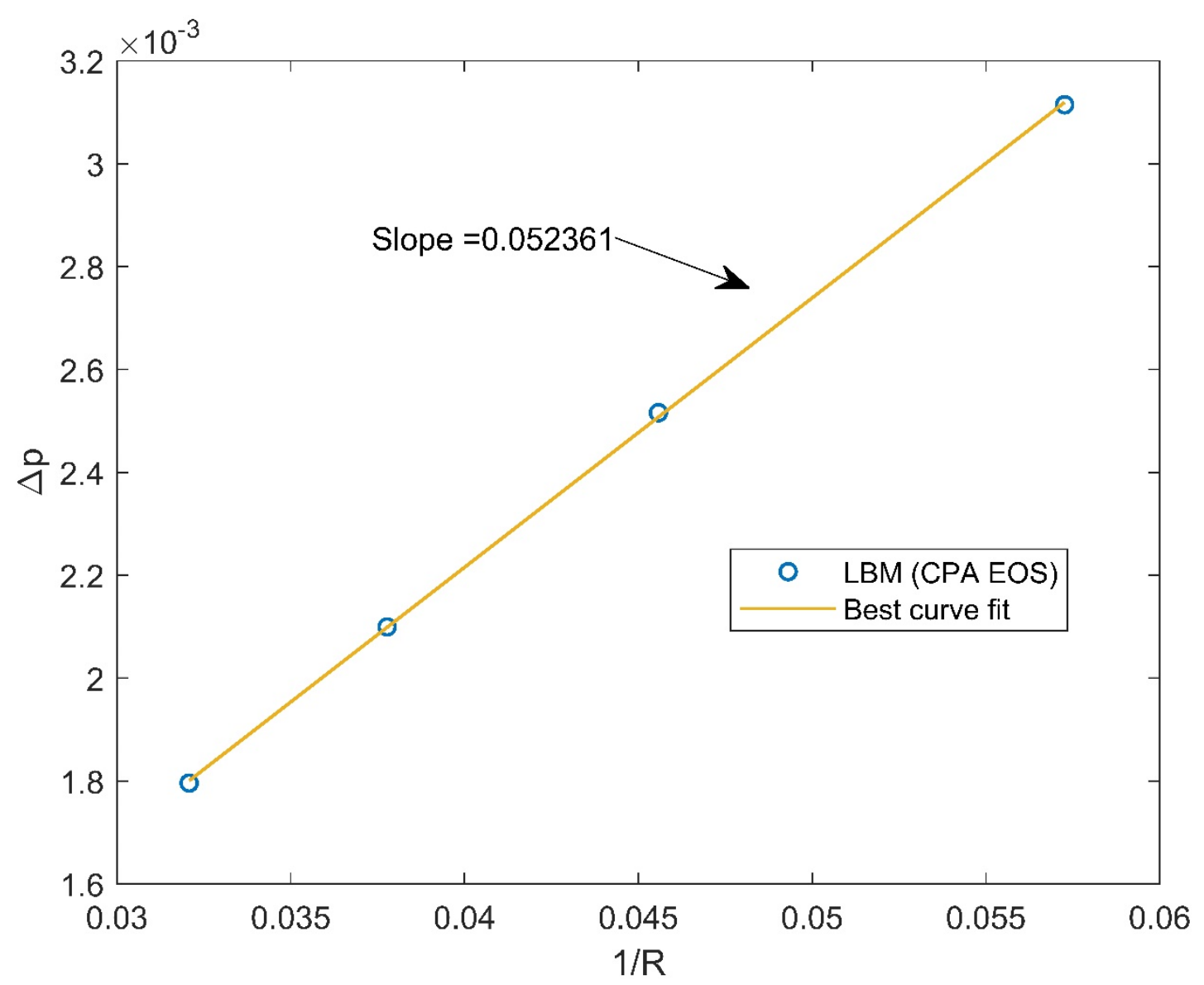

Figure 10: The pressure difference $\Delta p$ versus inverse radius of droplet $1 / R$.

Water is an important associating fluid in various biological, energy, and environment processes/systems. Accurate simulation of thermodynamic and physical properties of associating fluids including water can assist to better model, design, operate, and optimize the corresponding processes in terms of theoretical and practical prospects.

\section{SUMMERY AND CONCLUSIONS}

We introduce a pseudopotential central moments-based lattice Boltzmann method (LBM). Cubic plus association equation of state (CPA EOS) is implemented in the model to take into account the association forces in fluids with hydrogen bounds. The following main conclusions can be made based on the results of the current study: 
- Shan and Chen (SC) Sbragaglia and Shan (SS) EOSs do not have sufficient flexibility to predict the fluid phase behaviors. By changing their parameters their behaviour of predicting two phase densities do not change considerably (see Fig. 3).

- Double shear layer test shows that central moments-based LBM with a high-order Hermite polynomial are more stable than multiple-relaxation-time (MRT) collision operator-based LBM at turbulent cases. Thus, the central moments-based LBM is more suitable to be used for simulating high Reynolds and Mach number cases.

- Increasing the order of gradient operator will increase the stability of model in two-phase cases in terms of reduction of the spurious velocity.

- The gradient operator may lead to variations in the calculated interfacial tensions (capillary pressures) which can be manipulated by EOSs parameters.

- The type of the gradient operator may influence the thermodynamic consistency of the model.

- Observing a straight line in the capillary test can be considered as a proper approach for verifying the model.

The capability/potential of the current model to handle high-velocity cases and model the surface adsorption occurrence is an asset to effectively simulate transport phenomena in low permeable porous media. Further studies are recommended to investigate vital aspects such as computational costs, stability, and applicability ranges of various categories of LBM that can make easier implications of LBM in energy, environment, and health systems.

\section{CONFLICTS OF INTEREST}

There are no conflicts to declare.

\section{ACKNOWLEDGMENTS}

The authors would like to thank Memorial University, InnovateNL, and the Natural Sciences and Engineering Research Council of Canada (NSERC) for the financial assistance of this research project. 


\section{REFERENCES}

1. Aidun, C. K.; Clausen, J. R., Lattice-Boltzmann method for complex flows. Annual review of fluid mechanics 2010, 42, 439-472.

2. Chen, S.; Doolen, G. D., Lattice Boltzmann method for fluid flows. Annual review of fluid mechanics 1998, 30 (1), 329-364.

3. Succi, S., The lattice Boltzmann equation: for fluid dynamics and beyond. Oxford university press: 2001.

4. Succi, S.; Succi, S., The Lattice Boltzmann Equation: For Complex States of Flowing Matter. Oxford University Press: 2018.

5. Gupta, N.; Fathi, E.; Belyadi, F., Effects of nano-pore wall confinements on rarefied gas dynamics in organic rich shale reservoirs. Fuel 2018, 220, 120-129.

6. Tao, S.; Guo, Z., Boundary condition for lattice Boltzmann modeling of microscale gas flows with curved walls in the slip regime. Physical Review E 2015, 91 (4), 043305.

7. Yang, L.; Yu, Y.; Hou, G.; Wang, K.; Xiong, Y., Boundary conditions with adjustable slip length for the lattice Boltzmann simulation of liquid flow. Computers \& Fluids 2018, 174, $200-$ 212.

8. Abadi, R. H. H.; Fakhari, A.; Rahimian, M. H., Numerical simulation of three-component multiphase flows at high density and viscosity ratios using lattice Boltzmann methods. Physical Review E 2018, 97 (3), 033312.

9. Chen, L.; Kang, Q.; Mu, Y.; He, Y.-L.; Tao, W.-Q., A critical review of the pseudopotential multiphase lattice Boltzmann model: Methods and applications. International Journal of Heat and Mass Transfer 2014, 76, 210-236.

10. Li, Q.; Luo, K. H.; Kang, Q.; He, Y.; Chen, Q.; Liu, Q., Lattice Boltzmann methods for multiphase flow and phase-change heat transfer. Progress in Energy and Combustion Science 2016, 52, 62-105.

11. Liu, H.; Kang, Q.; Leonardi, C. R.; Schmieschek, S.; Narváez, A.; Jones, B. D.; Williams, J. R.; Valocchi, A. J.; Harting, J., Multiphase lattice Boltzmann simulations for porous media applications. Computational Geosciences 2016, 20 (4), 777-805.

12. He, Y.-L.; Liu, Q.; Li, Q.; Tao, W.-Q., Lattice Boltzmann methods for single-phase and solidliquid phase-change heat transfer in porous media: A review. International Journal of Heat and Mass Transfer 2019, 129, 160-197.

13. Arabjamaloei, R.; Ruth, D., Numerical study of inertial effects on permeability of porous media utilizing the Lattice Boltzmann Method. Journal of Natural Gas Science and Engineering 2017, 44, 22-36.

14. Chai, Z.; Shi, B.; Lu, J.; Guo, Z., Non-Darcy flow in disordered porous media: A lattice Boltzmann study. Computers \& Fluids 2010, 39 (10), 2069-2077.

15. Foroughi, S.; Jamshidi, S.; Pishvaie, M. R., New Correlative Models to Improve Prediction of Fracture Permeability and Inertial Resistance Coefficient. Transport in Porous Media 2018, 121 (3), 557-584. 
16. Hasert, M.; Bernsdorf, J.; Roller, S. In Lattice Boltzmann simulation of non-Darcy flow in porous media, ICCS, 2011; pp 1048-1057.

17. Sukop, M. C.; Huang, H.; Alvarez, P. F.; Variano, E. A.; Cunningham, K. J., Evaluation of permeability and non-Darcy flow in vuggy macroporous limestone aquifer samples with lattice Boltzmann methods. Water Resources Research 2013, 49 (1), 216-230.

18. Zhang, L.; Yang, S.; Zeng, Z.; Chew, J. W., Consistent boundary conditions of the multiplerelaxation-time lattice Boltzmann method for convection-diffusion equations. Computers \& Fluids 2018, 170, 24-40.

19. Wang, H.; Shi, B.; Liang, H.; Chai, Z., Finite-difference lattice Boltzmann model for nonlinear convection-diffusion equations. Applied Mathematics and Computation 2017, 309, 334-349.

20. Shi, B.; Guo, Z., Lattice Boltzmann simulation of some nonlinear convection-diffusion equations. Computers \& Mathematics with Applications 2011, 61 (12), 3443-3452.

21. Shi, B.; Guo, Z., Lattice Boltzmann model for nonlinear convection-diffusion equations. Physical Review E 2009, 79 (1), 016701.

22. Shi, B.; Deng, B.; Du, R.; Chen, X., A new scheme for source term in LBGK model for convection-diffusion equation. Computers \& Mathematics with Applications 2008, 55 (7), 15681575.

23. Lai, H.; Ma, C., A higher order lattice BGK model for simulating some nonlinear partial differential equations. Science in China Series G: Physics, Mechanics and Astronomy 2009, 52 (7), 1053-1061.

24. Chai, Z.; He, N.; Guo, Z.; Shi, B., Lattice Boltzmann model for high-order nonlinear partial differential equations. Physical Review E 2018, 97 (1), 013304.

25. Sukop, M., DT Thorne, Jr. Lattice Boltzmann Modeling Lattice Boltzmann Modeling. Springer: 2006.

26. Huang, H.; Sukop, M.; Lu, X., Multiphase lattice Boltzmann methods: Theory and application. John Wiley \& Sons: 2015.

27. Guo, Z.; Shu, C., Lattice Boltzmann method and its applications in engineering. World Scientific: 2013; Vol. 3.

28. Bhatnagar, P. L.; Gross, E. P.; Krook, M., A model for collision processes in gases. I. Small amplitude processes in charged and neutral one-component systems. Physical review 1954, 94 (3), 511.

29. Huang, H.; Krafczyk, M.; Lu, X., Forcing term in single-phase and Shan-Chen-type multiphase lattice Boltzmann models. Physical Review E 2011, 84 (4), 046710.

30. d'Humieres, D., Multiple-relaxation-time lattice Boltzmann models in three dimensions. Philosophical Transactions of the Royal Society of London. Series A: Mathematical, Physical and Engineering Sciences 2002, 360 (1792), 437-451.

31. Marié, S.; Ricot, D.; Sagaut, P., Comparison between lattice Boltzmann method and Navier-Stokes high order schemes for computational aeroacoustics. Journal of Computational Physics 2009, 228 (4), 1056-1070.

32. Lallemand, P.; Luo, L.-S., Theory of the lattice Boltzmann method: Dispersion, dissipation, isotropy, Galilean invariance, and stability. Physical Review E 2000, 61 (6), 6546.

33. Yu, Z.; Fan, L.-S., Multirelaxation-time interaction-potential-based lattice Boltzmann model for two-phase flow. Physical Review E 2010, 82 (4), 046708. 
34. Li, Q.; Luo, K.; Li, X., Lattice Boltzmann modeling of multiphase flows at large density ratio with an improved pseudopotential model. Physical Review E 2013, 87 (5), 053301.

35. Geier, M.; Greiner, A.; Korvink, J. G., Cascaded digital lattice Boltzmann automata for high Reynolds number flow. Physical Review E 2006, 73 (6), 066705.

36. Premnath, K. N.; Banerjee, S., On the three-dimensional central moment lattice Boltzmann method. Journal of Statistical Physics 2011, 143 (4), 747-794.

37. Geier, M.; Greiner, A.; Korvink, J. G., Properties of the cascaded lattice Boltzmann automaton. International Journal of Modern Physics C 2007, 18 (04), 455-462.

38. Asinari, P., Generalized local equilibrium in the cascaded lattice Boltzmann method. Physical Review E 2008, 78 (1), 016701.

39. Geier, M., De-aliasing and stabilization formalism of the cascaded lattice Boltzmann automaton for under-resolved high Reynolds number flow. International journal for numerical methods in fluids 2008, 56 (8), 1249-1254.

40. Geier, M.; Greiner, A.; Korvink, J. G., A factorized central moment lattice Boltzmann method. The European Physical Journal Special Topics 2009, 171 (1), 55-61.

41. Premnath, K. N.; Banerjee, S., Incorporating forcing terms in cascaded lattice Boltzmann approach by method of central moments. Physical Review E 2009, 80 (3), 036702.

42. Geier, M.; Schönherr, M.; Pasquali, A.; Krafczyk, M., The cumulant lattice Boltzmann equation in three dimensions: Theory and validation. Computers \& Mathematics with Applications 2015, 70 (4), 507-547.

43. De Rosis, A.; Lévêque, E., Central-moment lattice Boltzmann schemes with fixed and moving immersed boundaries. Computers \& Mathematics with Applications 2016, 72 (6), 16161628.

44. De Rosis, A., Alternative formulation to incorporate forcing terms in a lattice Boltzmann scheme with central moments. Physical Review E 2017, 95 (2), 023311.

45. Fei, L.; Luo, K. H., Consistent forcing scheme in the cascaded lattice Boltzmann method. Physical Review E 2017, 96 (5), 053307.

46. Geier, M.; Pasquali, A.; Schönherr, M., Parametrization of the cumulant lattice Boltzmann method for fourth order accurate diffusion part I: Derivation and validation. Journal of Computational Physics 2017, 348, 862-888.

47. Geier, M.; Pasquali, A.; Schönherr, M., Parametrization of the cumulant lattice Boltzmann method for fourth order accurate diffusion Part II: Application to flow around a sphere at drag crisis. Journal of Computational Physics 2017, 348, 889-898.

48. Kumar, C. S.; Mohankumar, S.; Geier, M.; Pattamatta, A., Numerical investigations on convective heat transfer enhancement in jet impingement due to the presence of porous media using cascaded lattice Boltzmann method. International Journal of Thermal Sciences 2017, 122, 201-217.

49. Shah, N.; Dhar, P.; Chinige, S. K.; Geier, M.; Pattamatta, A., Cascaded collision lattice Boltzmann model (CLBM) for simulating fluid and heat transport in porous media. Numerical Heat Transfer, Part B: Fundamentals 2017, 72 (3), 211-232.

50. Sharma, K. V.; Straka, R.; Tavares, F. W., New Cascaded Thermal Lattice Boltzmann Method for simulations of advection-diffusion and convective heat transfer. International Journal of Thermal Sciences 2017, 118, 259-277. 
51. Fei, L.; Luo, K. H., Cascaded lattice Boltzmann method for thermal flows on standard lattices. International Journal of Thermal Sciences 2018, 132, 368-377.

52. Fei, L.; Luo, K. H., Cascaded lattice Boltzmann method for incompressible thermal flows with heat sources and general thermal boundary conditions. Computers \& Fluids 2018, 165, 8995.

53. Fei, L.; Luo, K. H.; Li, Q., Three-dimensional cascaded lattice Boltzmann method: Improved implementation and consistent forcing scheme. Physical Review E 2018, 97 (5), 053309.

54. Geier, M.; Pasquali, A., Fourth order Galilean invariance for the lattice Boltzmann method. Computers \& Fluids 2018, 166, 139-151.

55. De Rosis, A., Nonorthogonal central-moments-based lattice Boltzmann scheme in three dimensions. Physical Review E 2017, 95 (1), 013310.

56. De Rosis, A.; Huang, R.; Coreixas, C., Universal formulation of central-moments-based lattice Boltzmann method with external forcing for the simulation of multiphysics phenomena. Physics of Fluids 2019, 31, 117102.

57. Grunau, D.; Chen, S.; Eggert, K., A lattice Boltzmann model for multiphase fluid flows. Physics of Fluids A: Fluid Dynamics 1993, 5 (10), 2557-2562.

58. Gunstensen, A. K.; Rothman, D. H.; Zaleski, S.; Zanetti, G., Lattice Boltzmann model of immiscible fluids. Physical Review A 1991, 43 (8), 4320.

59. Shan, X.; Chen, H., Simulation of nonideal gases and liquid-gas phase transitions by the lattice Boltzmann equation. Physical Review E 1994, 49 (4), 2941.

60. Shan, X.; Chen, H., Lattice Boltzmann model for simulating flows with multiple phases and components. Physical Review E 1993, 47 (3), 1815.

61. Swift, M. R.; Osborn, W.; Yeomans, J., Lattice Boltzmann simulation of nonideal fluids. Physical review letters 1995, 75 (5), 830.

62. Swift, M. R.; Orlandini, E.; Osborn, W.; Yeomans, J., Lattice Boltzmann simulations of liquid-gas and binary fluid systems. Physical Review E 1996, 54 (5), 5041.

63. He, X.; Chen, S.; Zhang, R., A lattice Boltzmann scheme for incompressible multiphase flow and its application in simulation of Rayleigh-Taylor instability. Journal of Computational Physics 1999, 152 (2), 642-663.

64. Liu, H.; Valocchi, A. J.; Kang, Q., Three-dimensional lattice Boltzmann model for immiscible two-phase flow simulations. Physical Review E 2012, 85 (4), 046309.

65. Sbragaglia, M.; Shan, X., Consistent pseudopotential interactions in lattice Boltzmann models. Physical Review E 2011, 84 (3), 036703.

66. Li, Q.; Luo, K. H.; Li, X., Forcing scheme in pseudopotential lattice Boltzmann model for multiphase flows. Physical Review E 2012, 86 (1), 016709.

67. Sbragaglia, M.; Benzi, R.; Biferale, L.; Succi, S.; Sugiyama, K.; Toschi, F., Generalized lattice Boltzmann method with multirange pseudopotential. Physical Review E 2007, 75 (2), 026702.

68. Falcucci, G.; Ubertini, S.; Succi, S., Lattice Boltzmann simulations of phase-separating flows at large density ratios: the case of doubly-attractive pseudo-potentials. Soft Matter 2010, $6(18), 4357-4365$.

69. Shan, X., Analysis and reduction of the spurious current in a class of multiphase lattice Boltzmann models. Physical Review E 2006, 73 (4), 047701.

70. Yuan, P.; Schaefer, L., Equations of state in a lattice Boltzmann model. Physics of Fluids 2006, 18 (4), 042101. 
71. Peng, D.-Y.; Robinson, D. B., A new two-constant equation of state. Industrial \& Engineering Chemistry Fundamentals 1976, 15 (1), 59-64.

72. Soave, G., Equilibrium constants from a modified Redlich-Kwong equation of state. Chemical engineering science 1972, 27 (6), 1197-1203.

73. Kontogeorgis, G. M.; Michelsen, M. L.; Folas, G. K.; Derawi, S.; von Solms, N.; Stenby, E. $\mathrm{H}$. , Ten years with the CPA (Cubic-Plus-Association) equation of state. Part 1. Pure compounds and self-associating systems. Industrial \& engineering chemistry research 2006, 45 (14), 48554868.

74. Asadi, M. B.; Zendehboudi, S., Hybridized method of pseudopotential lattice-Boltzmann and cubic-plus-association equations of state assess thermodynamic characteristics of associating fluids.

75. Kontogeorgis, G. M.; Voutsas, E. C.; Yakoumis, I. V.; Tassios, D. P., An equation of state for associating fluids. Industrial \& engineering chemistry research 1996, 35 (11), 4310-4318.

76. Wolfram, S., Cellular automaton fluids 1: Basic theory. Journal of statistical physics 1986, 45 (3-4), 471-526.

77. Shan, X., Pressure tensor calculation in a class of nonideal gas lattice Boltzmann models. Physical Review E 2008, 77 (6), 066702.

78. Benzi, R.; Biferale, L.; Sbragaglia, M.; Succi, S.; Toschi, F., Mesoscopic modeling of a twophase flow in the presence of boundaries: the contact angle. Physical Review E 2006, 74 (2), 021509.

79. Premnath, K. N.; Abraham, J., Three-dimensional multi-relaxation time (MRT) latticeBoltzmann models for multiphase flow. Journal of Computational Physics 2007, 224 (2), 539-559.

80. Lallemand, P.; Luo, L.-S., Theory of the lattice Boltzmann method: Acoustic and thermal properties in two and three dimensions. Physical review E 2003, 68 (3), 036706.

81. Malaspinas, O., Increasing stability and accuracy of the lattice Boltzmann scheme: recursivity and regularization. arXiv preprint arXiv:1505.06900 2015.

82. Coreixas, C.; Wissocq, G.; Puigt, G.; Boussuge, J.-F.; Sagaut, P., Recursive regularization step for high-order lattice Boltzmann methods. Physical Review E 2017, 96 (3), 033306.

83. Coreixas, C. G. High-order extension of the recursive regularized lattice Boltzmann method. 2018.

84. Huang, R.; Wu, H.; Adams, N. A., Eliminating cubic terms in the pseudopotential lattice Boltzmann model for multiphase flow. Physical Review E 2018, 97 (5), 053308.

85. Guo, Z.; Zheng, C.; Shi, B., Discrete lattice effects on the forcing term in the lattice Boltzmann method. Physical Review E 2002, 65 (4), 046308.

86. De Rosis, A.; Luo, K. H., Role of higher-order Hermite polynomials in the central-momentsbased lattice Boltzmann framework. Physical Review E 2019, 99 (1), 013301.

87. Lopez-Echeverry, J. S.; Reif-Acherman, S.; Araujo-Lopez, E., Peng-Robinson equation of state: 40 years through cubics. Fluid Phase Equilibria 2017, 447, 39-71.

88. Péneloux, A.; Rauzy, E.; Fréze, R., A consistent correction for Redlich-Kwong-Soave volumes. Fluid phase equilibria 1982, 8 (1), 7-23.

89. Firoozabadi, A., Thermodynamics and applications in hydrocarbon energy production. McGraw-Hill Education New York: 2016.

90. Huang, S. H.; Radosz, M., Equation of state for small, large, polydisperse, and associating molecules. Industrial \& Engineering Chemistry Research 1990, 29 (11), 2284-2294. 
91. Khajepor, S.; Wen, J.; Chen, B., Multipseudopotential interaction: a solution for thermodynamic inconsistency in pseudopotential lattice Boltzmann models. Physical Review $E$ 2015, 91 (2), 023301.

92. Colosqui, C. E.; Falcucci, G.; Ubertini, S.; Succi, S., Mesoscopic simulation of non-ideal fluids with self-tuning of the equation of state. Soft Matter 2012, 8 (14), 3798-3809.

93. Skuse, B.; Firoozabadi, A.; Ramey Jr, H. J., Computation and Interpretation of Capillary Pressure From a Centrifuge (includes associated papers 24962 and 25291). SPE formation evaluation 1992, 7 (01), 17-24.

94. Minion, M. L.; Brown, D. L., Performance of under-resolved two-dimensional incompressible flow simulations, II. Journal of Computational Physics 1997, 138 (2), 734-765.

95. Dellar, P. J., Lattice Boltzmann algorithms without cubic defects in Galilean invariance on standard lattices. Journal of Computational Physics 2014, 259, 270-283.

96. Mattila, K. K.; Philippi, P. C.; Hegele Jr, L. A., High-order regularization in lattice-Boltzmann equations. Physics of Fluids 2017, 29 (4), 046103.

97. Aulisa, E.; Manservisi, S.; Scardovelli, R., A novel representation of the surface tension force for two-phase flow with reduced spurious currents. Computer Methods in Applied Mechanics and Engineering 2006, 195 (44-47), 6239-6257.

98. Falcucci, G.; Bella, G.; Chiatti, G.; Chibbaro, S.; Sbragaglia, M.; Succi, S., Lattice Boltzmann models with mid-range interactions. Commun. Comput. Phys 2007, 2 (6), 1071-1084.

99. Falcucci, G.; Ubertini, S.; Biscarini, C.; Di Francesco, S.; Chiappini, D.; Palpacelli, S.; De Maio, A.; Succi, S., Lattice Boltzmann methods for multiphase flow simulations across scales. Communications in Computational Physics 2011, 9 (2), 269-296.

100. Mukherjee, S.; Berghout, P.; Van den Akker, H. E., A lattice Boltzmann approach to surfactant-laden emulsions. AIChE Journal 2018.

101. Trujillo-Pino, A.; Krissian, K.; Alemán-Flores, M.; Santana-Cedrés, D., Accurate subpixel edge location based on partial area effect. Image and Vision Computing 2013, 31 (1), 72-90. 